\title{
Minéralogie du puits de recherche plomb-zinc situé près de la ferme Erlenbach à Lontzen (Lontzen, Province de Liège)
}

Manuscrit reçu le 16 février 2018 et accepté le 21 mars 2018

Michel Blondieau ${ }^{1}$, Stéphane PuCcio ${ }^{2}$, Frédéric Hatert ${ }^{3}$

${ }^{1}$ Val des Cloches 131, B-6927 Tellin, Belgique

${ }^{2}$ Rue des Fontaines 156, B-4041 Vottem, Belgique

${ }^{3}$ Université de Liège, Laboratoire de Minéralogie B18, B-4000 Liège, Belgique

\section{Résumé}

Les travaux de recherches minières situés près de la ferme Erlenbach se trouvent dans le grand district $\mathrm{Pb}-\mathrm{Zn}$ de l'est de la Belgique à quelques kilomètres de La Calamine, le plus gros gisement métallique non ferreux belge. La minéralogie de ce petit gite non repris par Hatert et al. (2002) fait l'objet de cet article. On y recense un peu plus d'une vingtaine d'espèces minérales. Outre les minéraux classiques des chapeaux calaminaires, on y a observé deux espèces nouvelles pour la Belgique : la coronadite et le minium, ainsi qu'une autre espèce Al-Si-Zn-O apparemment nouvelle mais qui n'a pas pu être déterminée.

Mots clés : Gisement Pb-Zn, Erlenbach, district minier de Moresnet, Belgique, coronadite, minium.

\begin{abstract}
Small research workings for zinc ores occur near the Erlenbach farm not far from La Calamine, the biggest $\mathrm{Zn}-\mathrm{Pb}$ ore body in Belgium. This paper describes the minerals found in that locality, where two species are new for Belgium : minium and coronadite. Furthermore, an Al-Si-Zn-O mineral appears to be a new species but crystals found are far too tiny to authorize a description.
\end{abstract}

Keywords: Zinc ore, mineralogy, Belgium, Erlenbach, coronadite, minium.

\section{Introduction}

Le site étudié dans cet article, non signalé dans le livre "Les Minéraux de Belgique" (Hatert et al., 2002), est situé dans la commune de Lontzen, à proximité de la ferme dite "Erlenbach". Polrot (2002) écrit : "Le site est appelé Elsenbach sur la carte Vieille-Montagne 1894 et sur le plan Cadastral mais Erlenbach sur la carte allemande et les cartes de l'I.G.N." A proximité, un autre petit site minier porte aussi le nom d'Elsenbach, ce qui a conduit à de nombreuses confusions qui perdurent encore (Figure 1). 


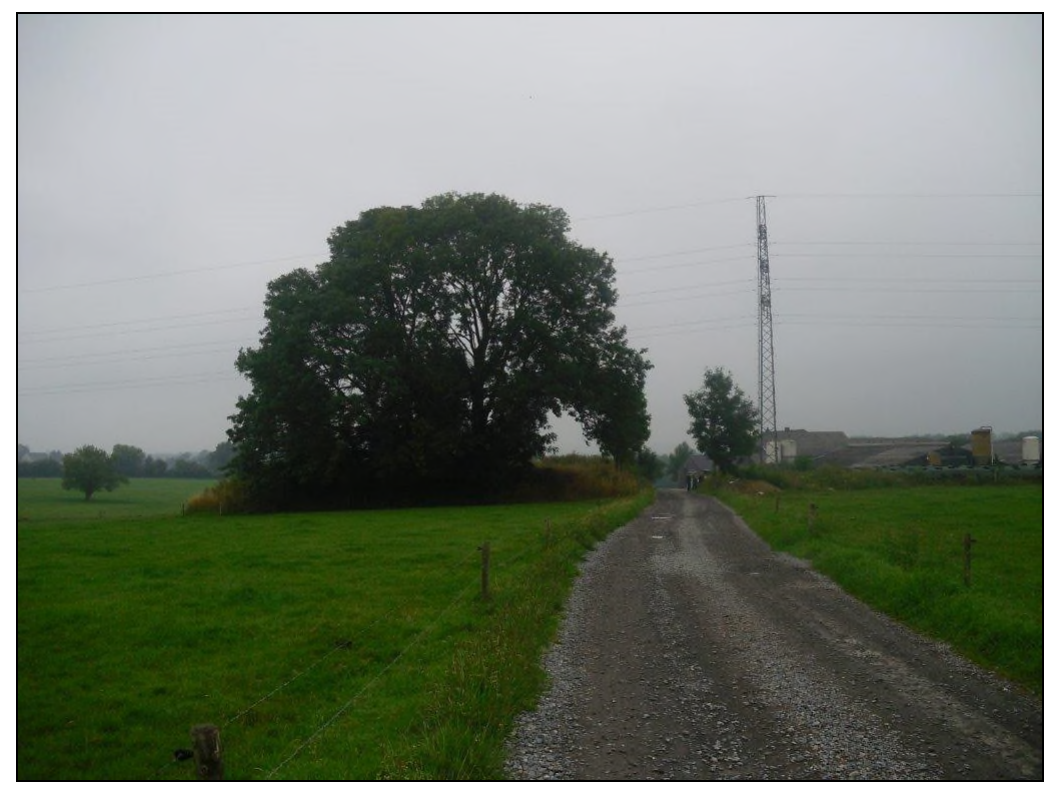

Fig.1 : Haldes d'Erlenbach à demi camouflées derrière un gros arbre, le long du chemin qui conduit à la ferme. (Photo M. Meunier, 2015).

Il y a quelques années, il était encore possible de voir au milieu des haldes un trou en entonnoir qui marquait l'emplacement du puits et autour duquel se trouvaient deux gros blocs de béton, anciens témoins des infrastructures qui l'équipaient. Les haldes ont été aplanies il y a quelques années, sans doute en 2014, et le trou en entonnoir rebouché est surmonté d'une dalle en béton sur laquelle se trouve une plaque avec pour seule indication : "Puits de mine N²52097" (Figure 2).

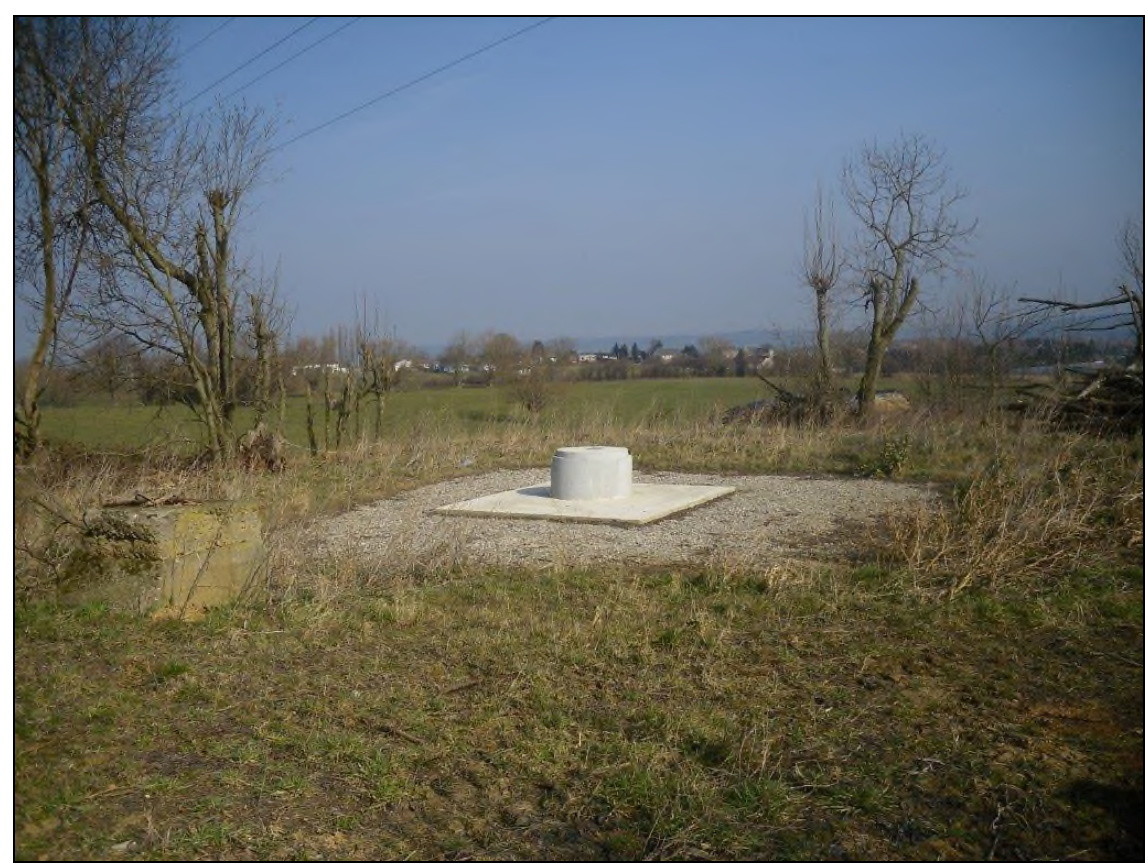

Fig. 2 : Puits d'Erlenbach sur les haldes aplanies. Sur la gauche, gros bloc de béton qui supportait les infrastructures du puits. (Photo M. Meunier, 2015). 
On connaît finalement peu de chose de l'histoire minière de ces travaux. Dans le document de renonciation de la concession de la Vieille-Montagne établi par "Union Minière" (2000), on signale des travaux d'approche par galerie depuis Eschbroich (à -132 m) entrepris par la Société de la "Vieille-Montagne" afin de vérifier la continuité vers le sud-ouest des amas minéralisés comme ceux observés à Stinkert. Ces travaux ont malheureusement été arrêtés prématurément à environ $250 \mathrm{~m}$ du puits d'Erlenbach, lors de la fermeture de la mine de Schmalgraf en 1932.

Polrot (2002) indique que les allemands auraient fait des travaux sur le gisement pendant la deuxième guerre mondiale. À ce propos, le site «Mindat» (page Erlenbach, février 2017) reprend les notes d'un certain Jean Xhonneux qui exploitait la ferme à cette époque : " ... excavations were started in November 1940 by the "Stolberger Zinc Works". 20-30 miners were working in three shifts. [...] In the first year, the shaft had reached a depth of 15 meters. At this level a bit of blende was mined. The detected ore was found as a layer having a thickness of $15 \mathrm{~cm}$, the layer was a meter wide and 15 meters long. After further drilling in 1941 to a depth of $35 \mathrm{~m}$..."

Selon cette source, le puits aurait donc atteint une profondeur de 35 mètres et aurait recoupé une petite lentille de blende (sphalérite rubanée). C'était un minerai activement exploité dans la région notamment à l'importante mine voisine de Schmalgraf qui en a produit plus de 330.000 tonnes lavées et triées (Dejonghe et al., 1993). Sa présence à Erlenbach est fort possible mais on n'en retrouve pourtant que fort peu aujourd'hui dans les haldes, alors qu'on y observe en abondance des « calamines » qui, elles, n'ont pas été signalées par Xhonneux.

\section{Contexte géologique}

Erlenbach se trouve dans le grand district minier $\mathrm{Pb}-\mathrm{Zn}$ de l'est de la Belgique et est inclus dans le périmètre de la concession de la "Vieille-Montagne", à peu de distance des mines de La Calamine (Altenberg) et de Schmalgraf (Figure 3). 


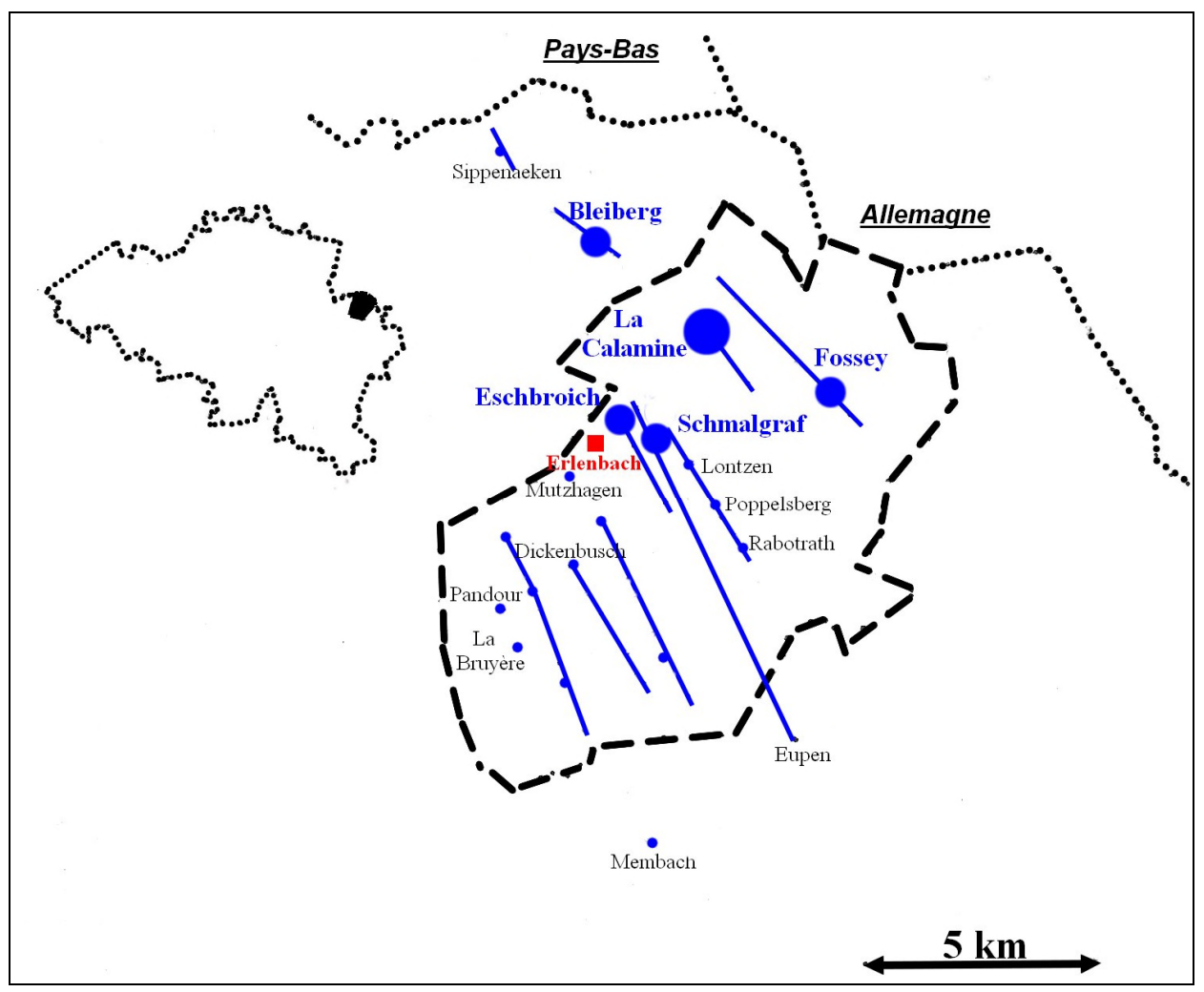

Fig. 3 : Localisation d'Erlenbach dans la concession "Vieille-Montagne". Les failles minéralisées et les principaux sièges de production sont indiqués en bleu. Le filon de Bleiberg et les amas associés ne faisaient pas partie de cette concession (d'après Dejonghe \& Ladeuze, 1994, modifié).

Le site minier d'Erlenbach se trouve sur la bordure nord du synclinal de Schmalgraf et, comme à Stinkert et à Eschbroich, il présente un peu de minerai dans la zone de contact lithostratigraphique entre le Viséen et le Tournaisien, directement sous la couverture postpaléozoïque (Figures 4 et 5). Dans la région, seules les failles transversales aux couches sont minéralisées. Les filons peuvent cependant s'épancher en amas importants lorsqu'ils passent du namurien détritique au calcaire viséen ; ce sont les fameux "amas de contact lithostratigraphique" qui ont produit les plus grands tonnages de minerai. On en a notamment observé à Schmalgraf et à Eschbroich. Des amas entre les calcaires viséens et les calcaires tournaisiens sont également observés. Mais plus on s'éloigne de la faille transversale où est logé le filon nourricier, plus ces amas sont petits. Le site d'Erlenbach est déjà fort éloigné de la faille minéralisée où est logé le filon d'Eschbroich, ce qui rend compte du faible développement probable de cet amas de contact lithostratigraphique. 


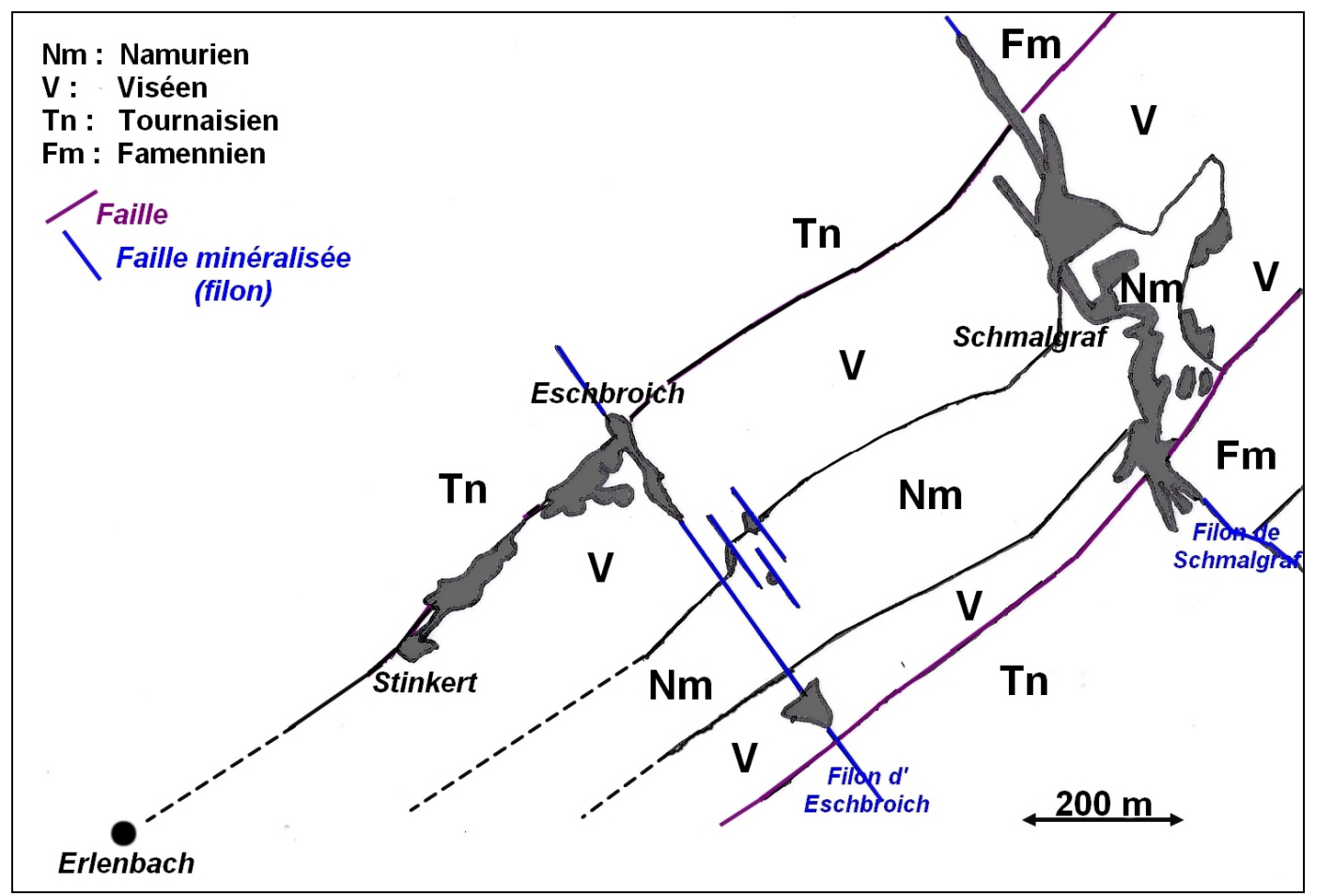

Fig. 4 : Carte géologique simplifiée sous la couverture postpaléozoïque, montrant la position d'Erlenbach au nord d'un synclinal à cœur namurien d'axe NE-SW. Les parties hachurées correspondent aux poches de minerai exploitées aux mines de Schmalgraf, Eschbroich et Stinkert. Les minéralisations d'Erlenbach sont, comme à Stinkert, des amas de contact lithostratigraphique Viséen/Tournaisien (d'après Dejonghe et al., 1993, modifié).

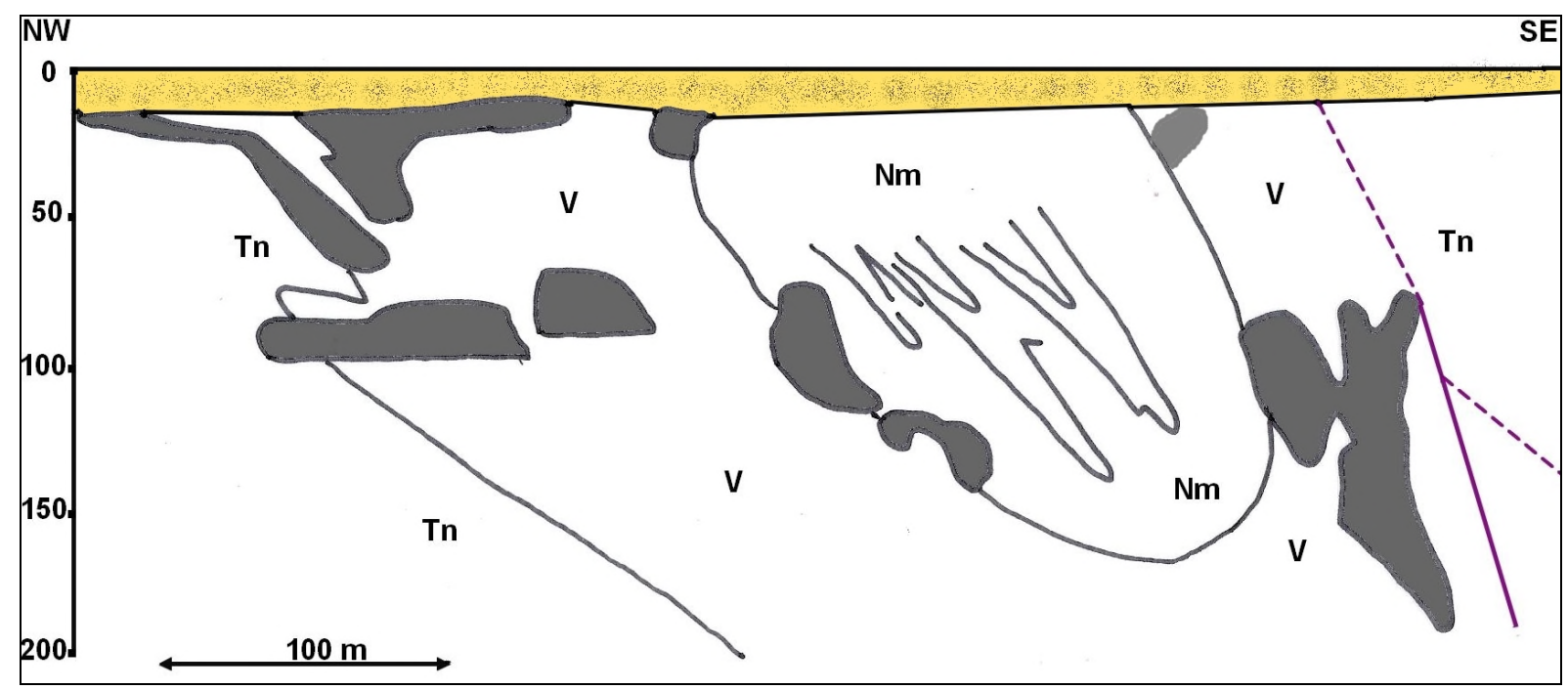

Fig. 5 : Coupe NW-SE selon la faille minéralisée d'Eschbroich (d'après Dejonghe et al., 1993, modifié). En plus des amas lithostratigraphiques profonds (exploitation jusqu'à-182 mètres à Eschbroich), on observe également des amas directement sous la couverture sablo-argileuse postpaléozoïque (en jaune), comme cela a sans doute été le cas aussi à Erlenbach. 


\section{Descriptions minéralogiques}

La minéralogie du site est étudiée sur des échantillons que nous avons récoltés sur près de trente ans dans les déblais miniers. Les blocs minéralisés sont disséminés dans une argile brun rouge extrêmement collante, dans laquelle se trouvent des rognons de galène très altérée ainsi que de la smithsonite du type «calamine roche ». La limonite plus ou moins pulvérulente est fréquente et est souvent incrustée de cristaux millimétriques de cérusite bacillaire blanche. Parfois, les blocs à limonite passent à une sorte de brèche rouge avec nombreux fragments de quartz associés à de la cérusite. Parfois aussi, on observe une argile gris bleuté dans laquelle se trouvent de rares morceaux de smithsonite de même couleur et couverte de minuscules cristaux uniquement observables à la loupe. Hormis la galène, les sulfures (marcasite et sphalérite rubanée) sont relativement rares dans les déblais.

Les minéraux ont été soumis à une analyse par diffraction des rayons $\mathrm{X}$ sur un diffractomètre de poudres PANALYTICAL PW-3710 utilisant la radiation $\operatorname{FeK}_{\alpha}(\lambda=1,9373 \AA$ ). Lorsque les quantités de matière étaient très faibles, un diffractomètre à 4 cercles Rigaku Xcalibur, équipé d'un détecteur EOS et utilisant la radiation $\operatorname{MoK}_{\alpha}(\lambda=0,7107 \AA)$, a été utilisé en mode «Debye-Scherrer ». La morphologie des minéraux a été observée à l'aide d'un microscope électronique à balayage environnemental FEI ESEM-FEG XL 30 (CAREM-ULg, Cellule d'Appui à la Recherche et à l'Enseignement en Microscopie de l'Université de Liège). Les images ont été obtenues en électrons secondaires (mode «haut-vide») sous une tension d'accélération de $15 \mathrm{kV}$ et sur des échantillons métallisés au platine. Des spectres EDS («Energy Dispersive X-ray Spectrometry ») de microanalyse élémentaire aux rayons X ont été obtenus avec le même microscope, équipé d'un détecteur de rayons X Si(Li) EDAX ou SDD Bruker $129 \mathrm{eV}$. Selon les cas, la tension d'accélération était réglée à 15, 20 ou $30 \mathrm{kV}$. Les spectres ont été traités soit par le logiciel EDAX, soit par le logiciel Quanta Esprit 1.9 qui a fourni l'identification des éléments. 


\subsection{Allophane, $\mathrm{Al}_{2} \mathrm{O}_{3}\left(\mathrm{SiO}_{2}\right)_{1,3-2,0} \cdot 2,5-3, \mathrm{OH}_{2} \mathrm{O}$}

Un minéral vitreux incolore, blanc bleuté (Figure 6) ou teinté en brun, fréquemment associé à la dundasite et à la cérusite, est reconnu visuellement comme étant de l'allophane. Une esquille incolore analysée par diffraction des rayons X confirme l'identification du minéral. L'allophane est une espèce fragile mais assez régulièrement rencontrée dans les gisements $\mathrm{Pb}$ Zn de l'est de la Belgique. Elle est notamment signalée à Oneux, Bleiberg et La Calamine (Hatert et al., 2002), et nous l'avons aussi observée à Rabotrath (Blondieau et al., 2016).

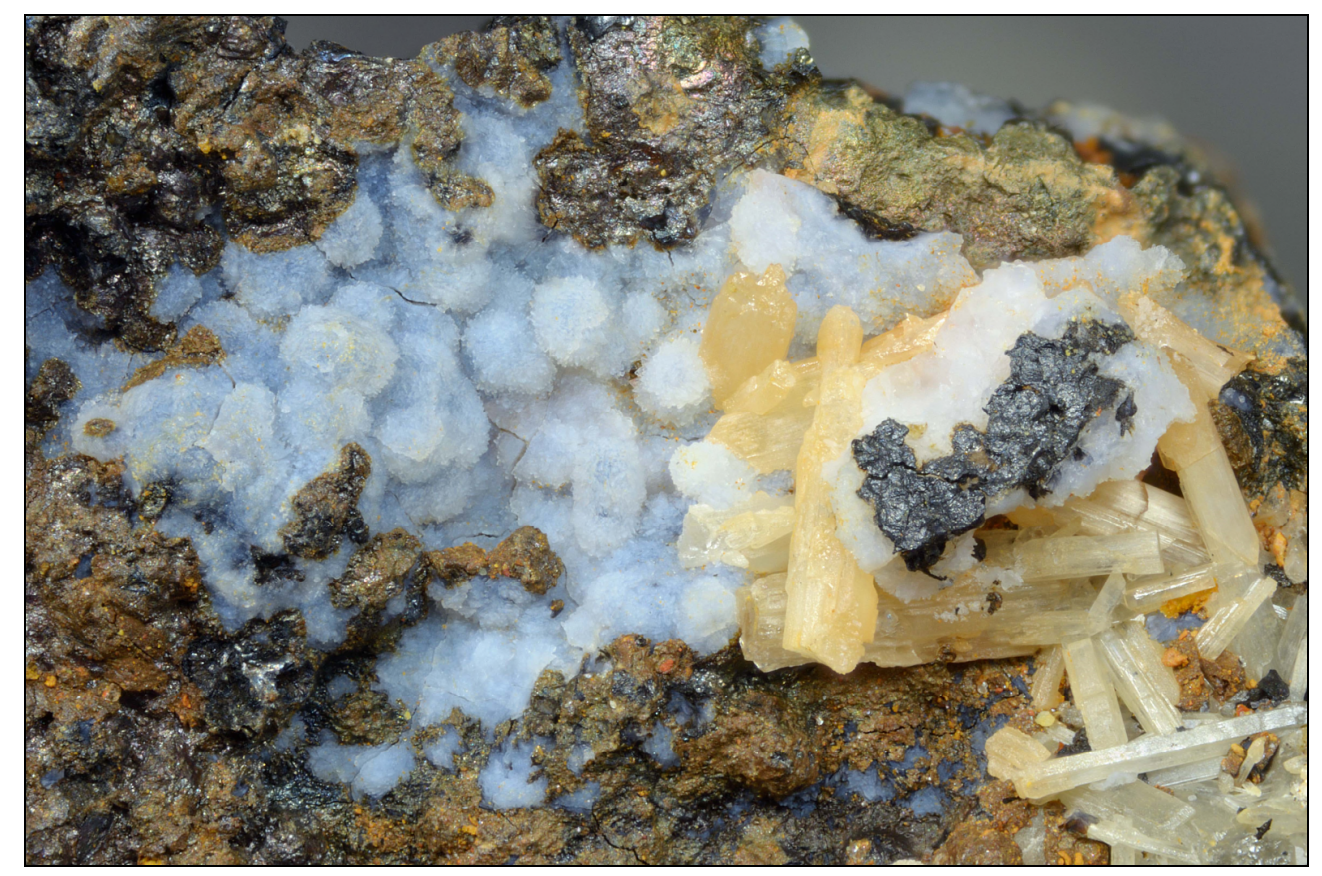

Fig. 6 : Allophane bleutée et cérusite blanche d'Erlenbach. $\mathrm{L}=4,25 \mathrm{~mm}$. (Photo P. Chollet).

\subsection{Anglésite, $\mathrm{PbSO}_{4}$}

L'anglésite est très abondante à Erlenbach. Associée à la cérusite, elle forme des encroûtements gris blanchâtres autour des nodules de galène (Figure 7). Parfois, la transformation est complète, et des nodules sont alors constitués d'anglésite pure montrant, dans des petites cavités, des cristaux prismatiques lumineux incolores ou gris de taille millimétrique. L'identification du minéral a été confirmée par diffraction des rayons $\mathrm{X}$. Les cristaux, au plus millimétriques, peuvent se présenter sous différents habitus (Figure 8). 
Bulletin de la Société des Sciences de Liège, Vol. 87, articles, 2018, p. 22 - 55

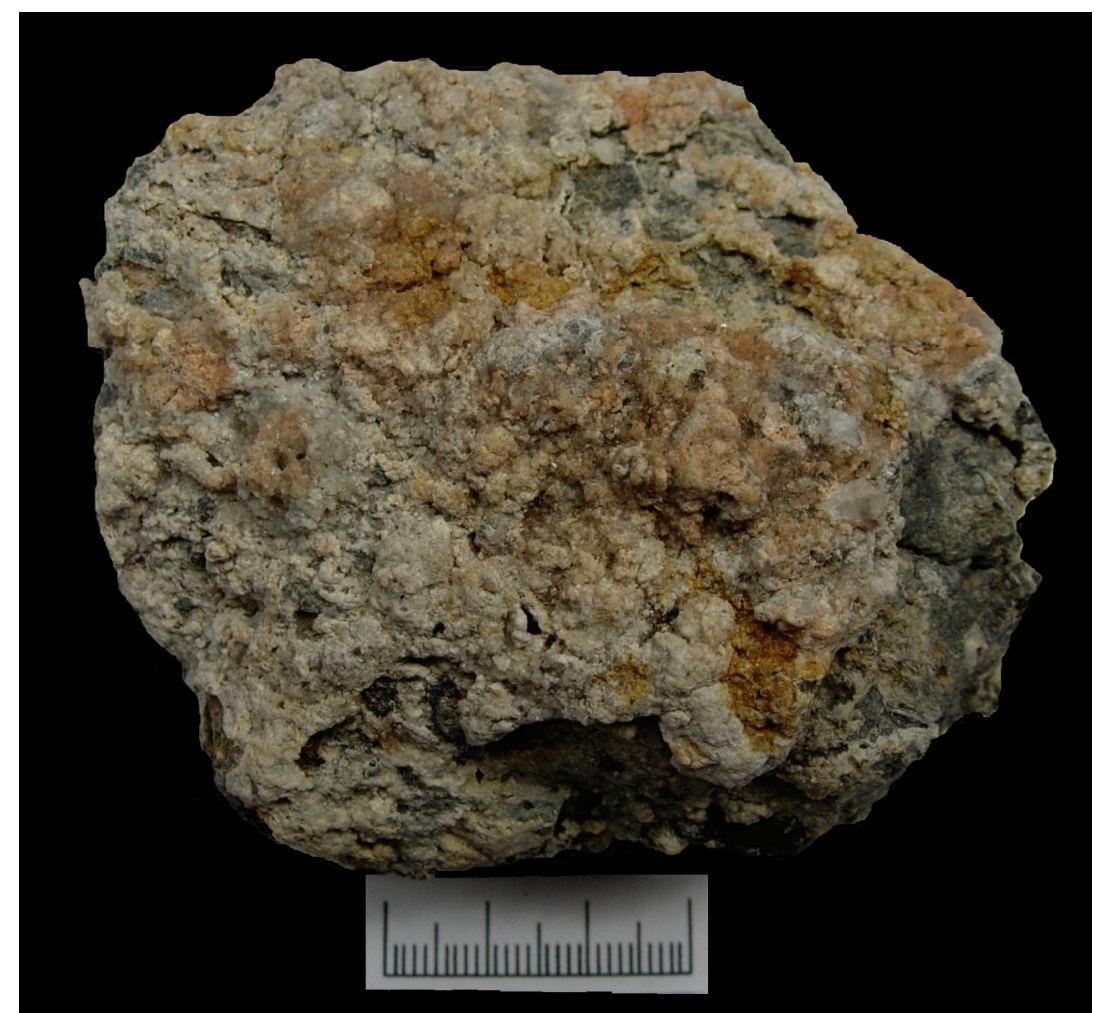

Fig. 7 : Nodule de galène couvert d'anglésite, Erlenbach. (Coll. et Photo : M. Blondieau).
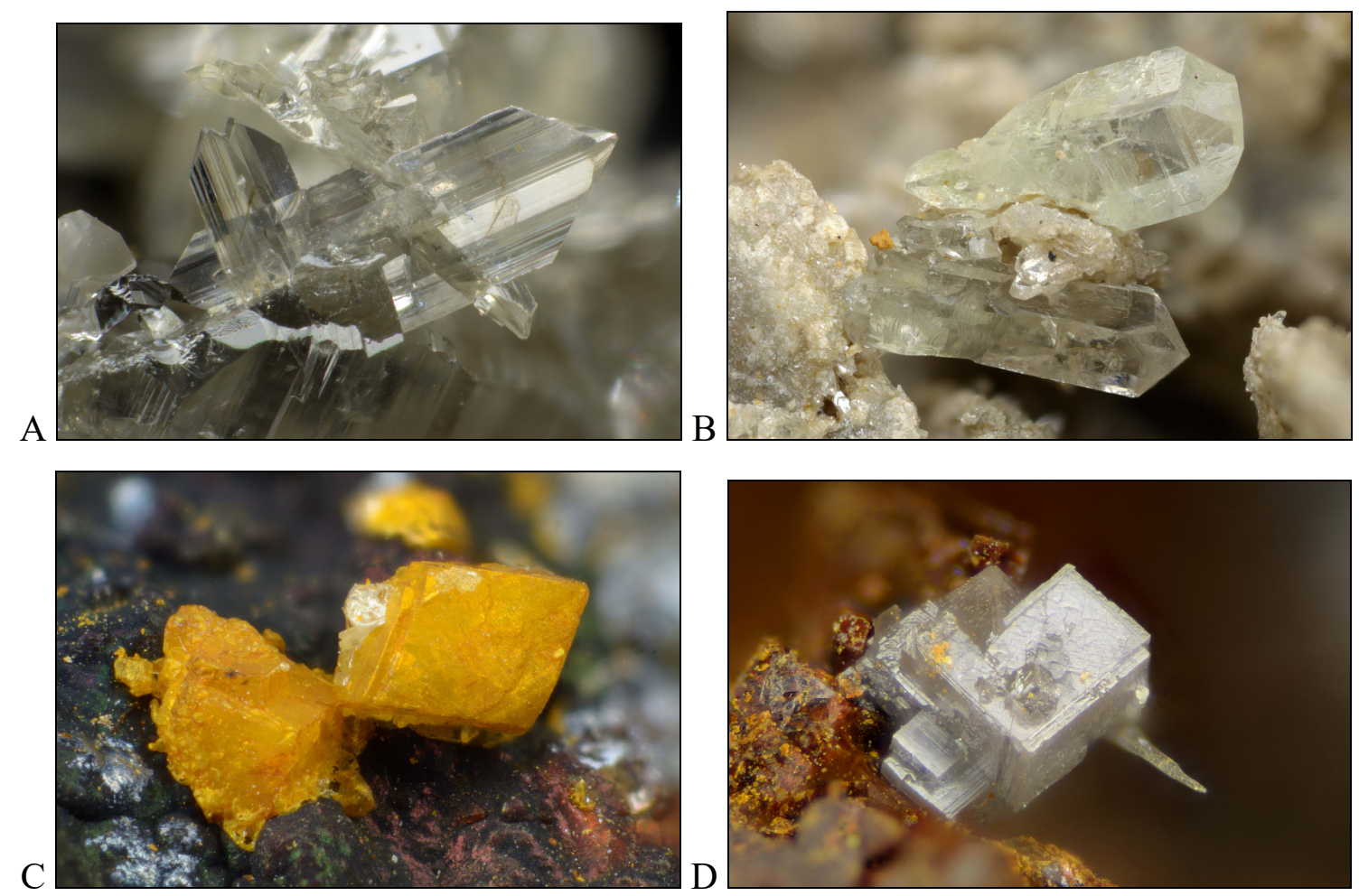

Fig. 8 : Anglésite d'Erlenbach sous différents habitus. $L=1,25 \mathrm{~mm}(A), L=2,35 \mathrm{~mm}(B)$, $\mathrm{L}=1,25 \mathrm{~mm}(\mathrm{C}), \mathrm{L}=0,85 \mathrm{~mm}(\mathrm{D})$. (Photos P. Chollet). 


\subsection{Barite, $\mathrm{BaSO}_{4}$}

Une tablette blanchâtre "en losange" de deux millimètres, ainsi que de plus petites tablettes incolores, ont été observées sur un seul échantillon, dans une cavité de blende rubanée. Une analyse par diffraction des rayons $\mathrm{X}$ a indiqué qu'il s'agit de barite, une espèce peu fréquente dans la zone métallifère autour de La Calamine, où elle n'est signalée que dans le filon de Bleiberg à Plombières, en aiguilles jaunâtres allongées (Hatert et al., 2002).

\subsection{Cérusite, $\mathrm{PbCO}_{3}$}

La cérusite est très abondante à Erlenbach. On l'observe à la surface de fragments de la brèche rouge ou dans des petites cavités de limonite/goethite (Figure 9). Elle se présente en cristaux blancs souvent très fins pouvant atteindre une longueur centimétrique, parfois recouverts d'une pellicule de goethite (Figure 10). Dans certaines parties de la brèche, elle forme également le ciment liant de minuscules fragments de quartz. L'identification du minéral est confirmée par diffraction des rayons X.

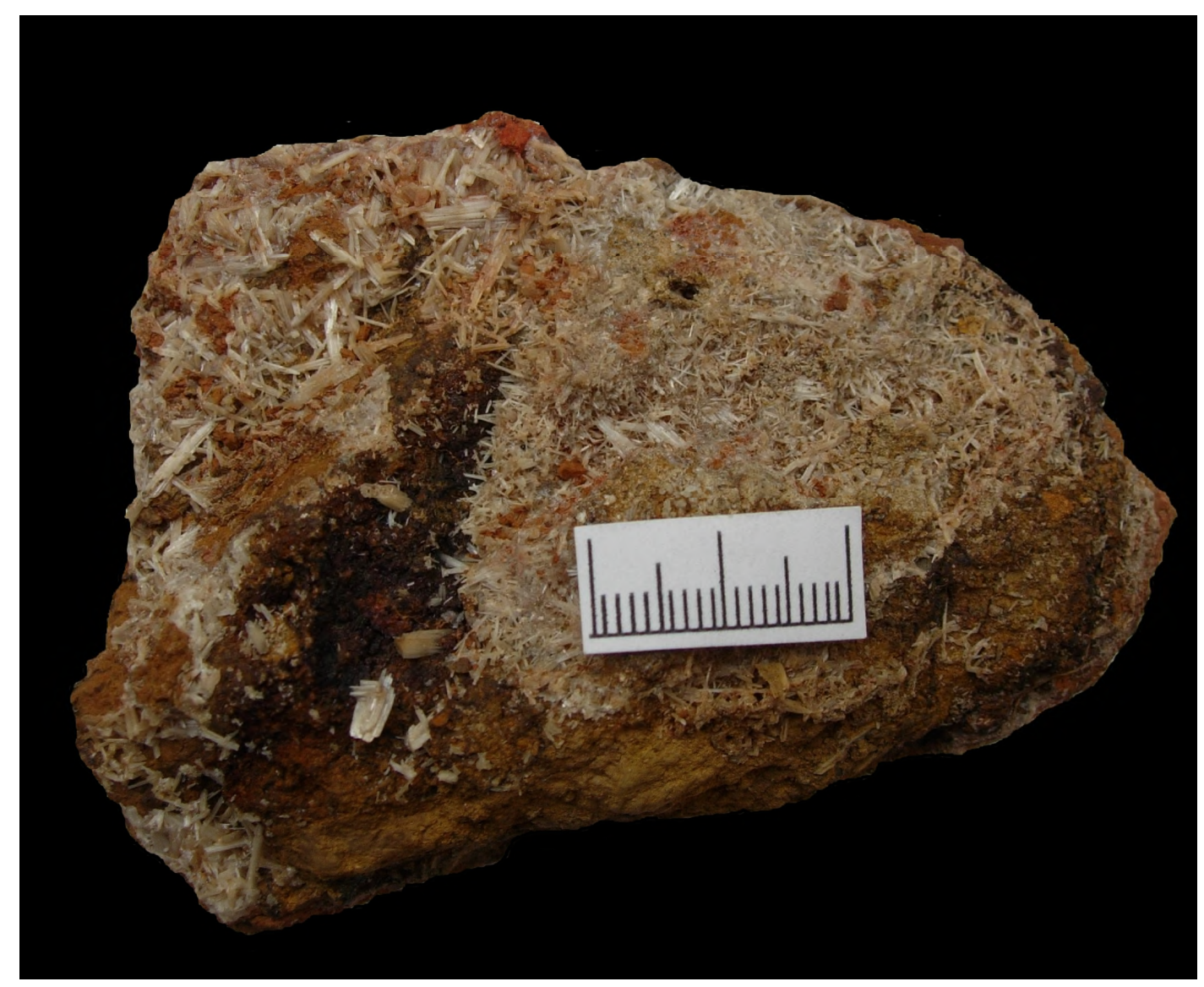

Fig. 9 : Cérusite en petits cristaux bacillaires sur limonite, Erlenbach. (Coll. et Photo : M. Blondieau). 
Bulletin de la Société des Sciences de Liège, Vol. 87, articles, 2018, p. 22 - 55
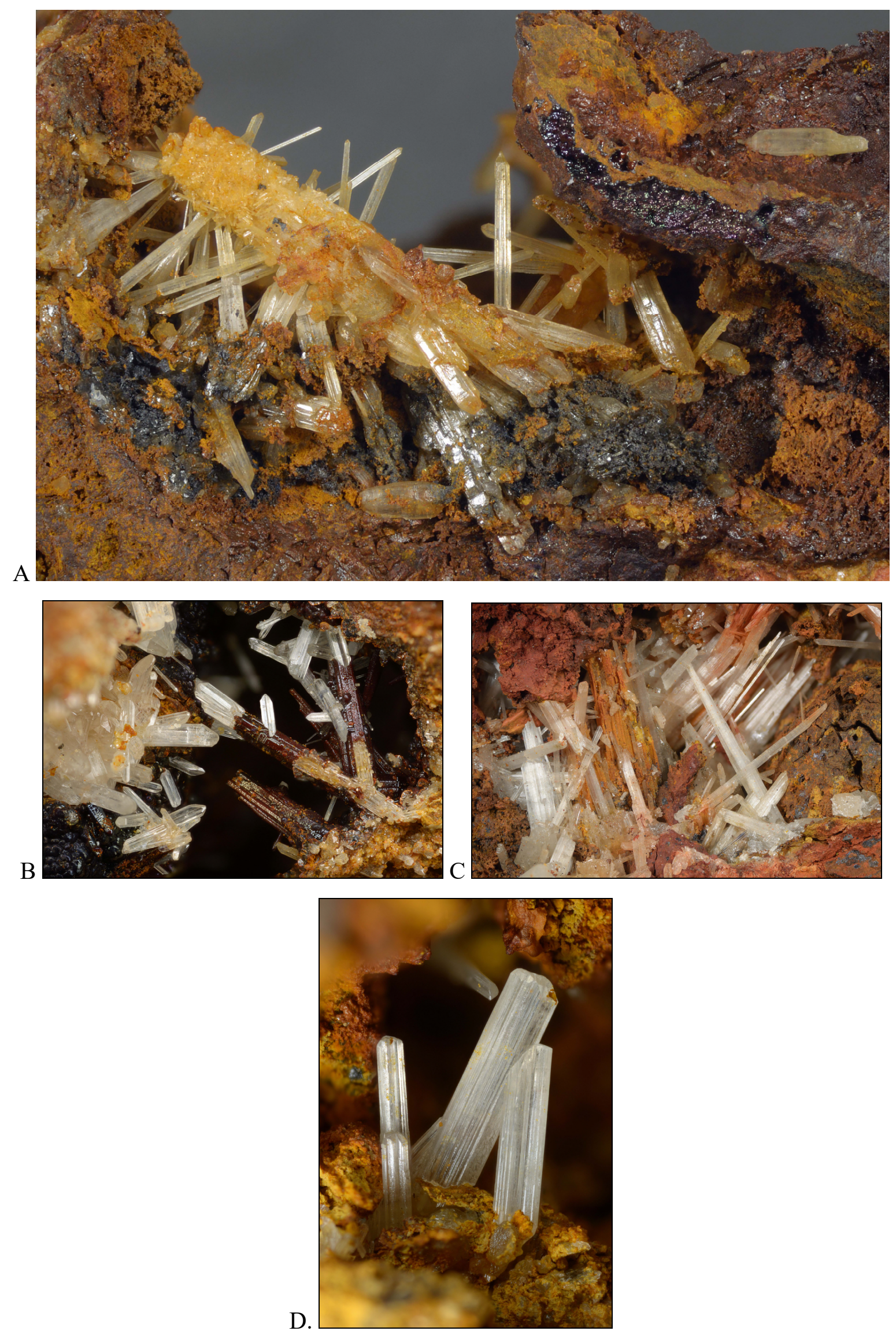

Fig. 10 : Cérusite d'Erlenbach sur limonite. $\mathrm{L}=7 \mathrm{~mm}(\mathrm{~A}), \mathrm{L}=3,1 \mathrm{~mm}(\mathrm{~B}), \mathrm{L}=9,1 \mathrm{~mm}(\mathrm{C})$, $\mathrm{L}=2,9 \mathrm{~mm}$ (D). (Photos P. Chollet). 


\subsection{Chalcophanite, $\mathrm{ZnMn}^{4+}{ }_{3} \mathrm{O}_{7} \cdot 3 \mathrm{H}_{2} \mathrm{O}$}

Comme à Rabotrath (Blondieau et al., 2016), la chalcophanite est fréquemment associée aux cristaux de smithsonite et peut se présenter en croûtes terreuses fragiles de couleur brune, avec des reflets violacés et une texture fibreuse sur cassure (Figures 11a et 11d). Les croûtes fragiles présentent en surface de minuscules cristaux (Figures $11 \mathrm{~b}$ et 11c). La chalcophanite est identifiée par diffraction des rayons $\mathrm{X}$ ainsi que par des analyses chimiques qualitatives EDS qui mettent en évidence les éléments majoritaires Mn, $\mathrm{Zn}$ et $\mathrm{O}$.

A.
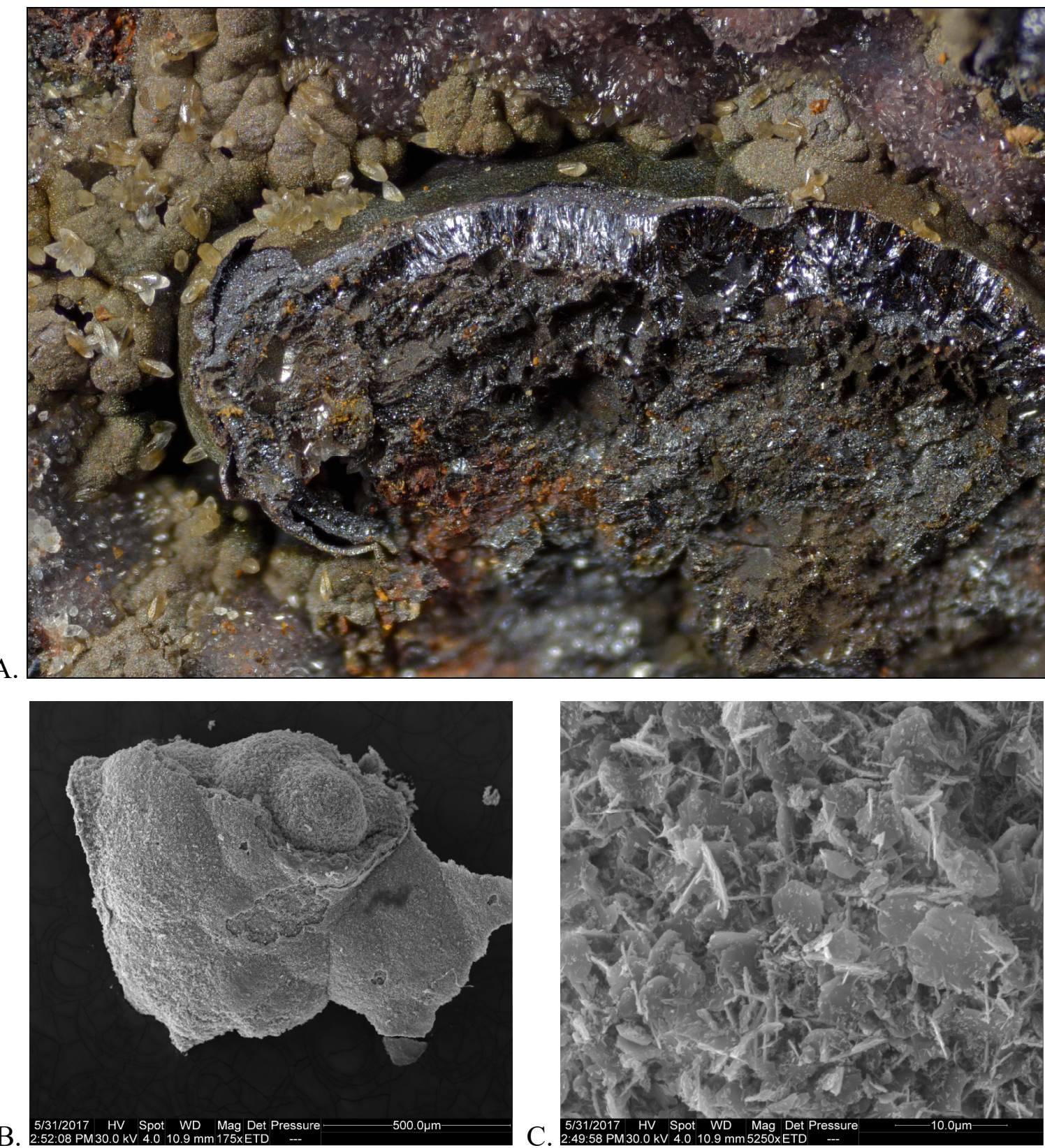


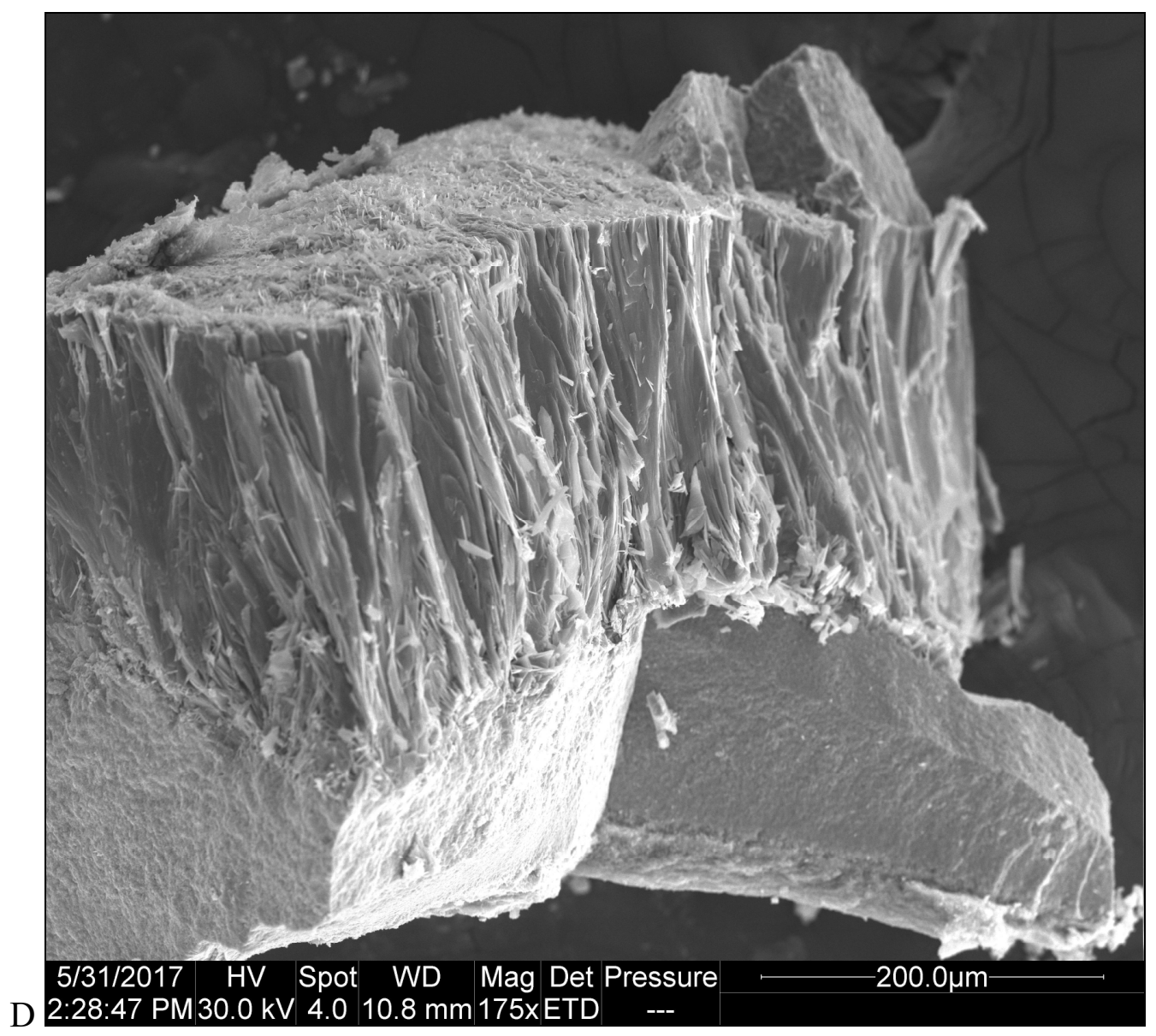

Fig. 11 : (A) Chalcophanite d'Erlenbach associée à de minuscules cristaux de smithsonite. $\mathrm{L}=2,7 \mathrm{~mm}$, (Coll. M. Blondieau, Photo P. Chollet). (B, C et D) Photographies au microscope électronique à balayage. (B et $\mathrm{C}$ ) Échantillon ERL 02c. Aspect d'une croûte de chalcophanite (B) et gros plan sur la structure de surface (C). (D) Échantillon ERL 02a. Chalcophanite fibreuse sur coronadite.

\subsection{Coronadite, $\mathrm{Pb}\left(\mathrm{Mn}^{4+}{ }_{6} \mathrm{Mn}^{3+}{ }_{2}\right) \mathrm{O}_{16}$}

Des petites sphérules couvertes en surface d'une matière fibreuse montrent sur cassure une partie interne visuellement plus dense, avec un éclat submétallique gris acier (Figure 12). L'analyse par diffraction des rayons $\mathrm{X}$, réalisée sur ces boules, indique la présence d'un mélange de chalcophanite et de coronadite. Cette dernière espèce montre un pic (100) à 3,124 Å, qui n'est pas présent dans la chalcophanite.

L'analyse chimique qualitative par EDS confirme l'association de ces deux espèces. La partie fibreuse est constituée des éléments majoritaires $\mathrm{Mn}, \mathrm{Zn}$ et $\mathrm{O}$ : il s'agit de chalcophanite. La partie interne plus massive montre la présence des éléments $\mathrm{Mn}, \mathrm{Pb}$ et $\mathrm{O}$ : il s'agit de coronadite. La coronadite est une espèce nouvelle pour la Belgique. 


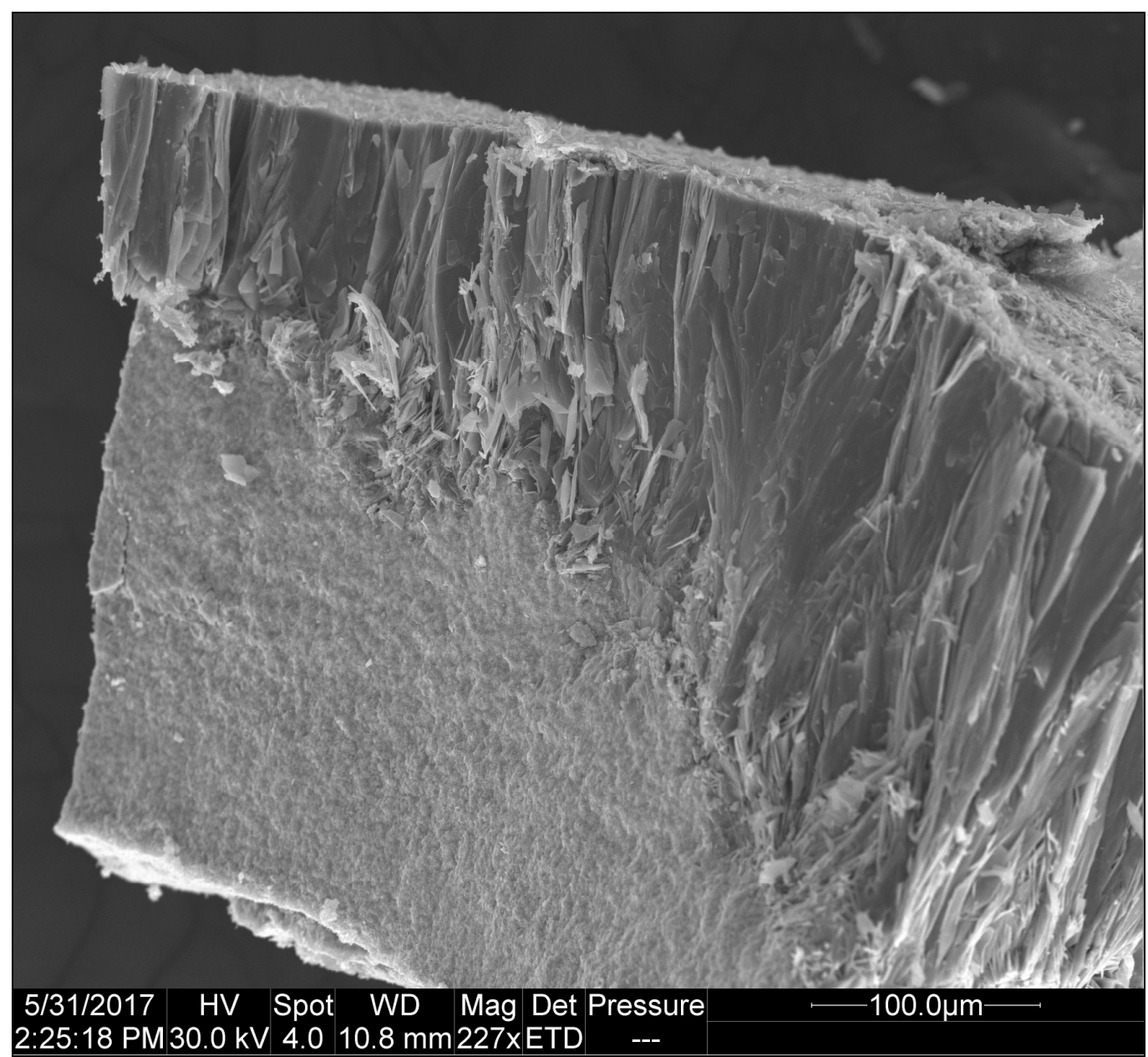

Fig 12 : Chalcophanite fibreuse sur coronadite massive, Erlenbach. Échantillon ERL 2a. Photographie au microscope électronique à balayage, mode électrons secondaires.

\subsection{Covellite, CuS}

De minuscules efflorescences avec éclat métallique bleu acier sont observées dans des cavités d'un échantillon de galène, ainsi que dans une fissure d'un échantillon de blende zonée. Le minéral est visuellement comparable à la covellite de postformation de Masbourg (Blondieau et al., 2017) et cette identification est confortée par les analyses chimiques qualitatives faites par EDS qui mettent bien en évidence la seule présence des éléments $\mathrm{Cu}$ et $\mathrm{S}$. Le diffractogramme de poudres de rayons $\mathrm{X}$ obtenu, bien que montrant également des pics importants de l'anglésite, confirme également l'identification de la covellite. En microscopie électronique, le minéral montre de minuscules cristaux tabulaires d'aspect hexagonal assez caractéristiques de l'espèce, ici regroupés en rosettes (Figure 13). Le gros cristal observé au centre de cette photographie est de l'anglésite. Cette association d'anglésite et de covellite sur galène est assez habituelle dans la zone de cémentation, lorsque des solutions chargées en 
sulfate de cuivre arrivent en contact avec de la galène. Il se forme alors de l'anglésite $\left(\mathrm{PbSO}_{4}\right)$ et de la covelline ( $\mathrm{CuS})$.

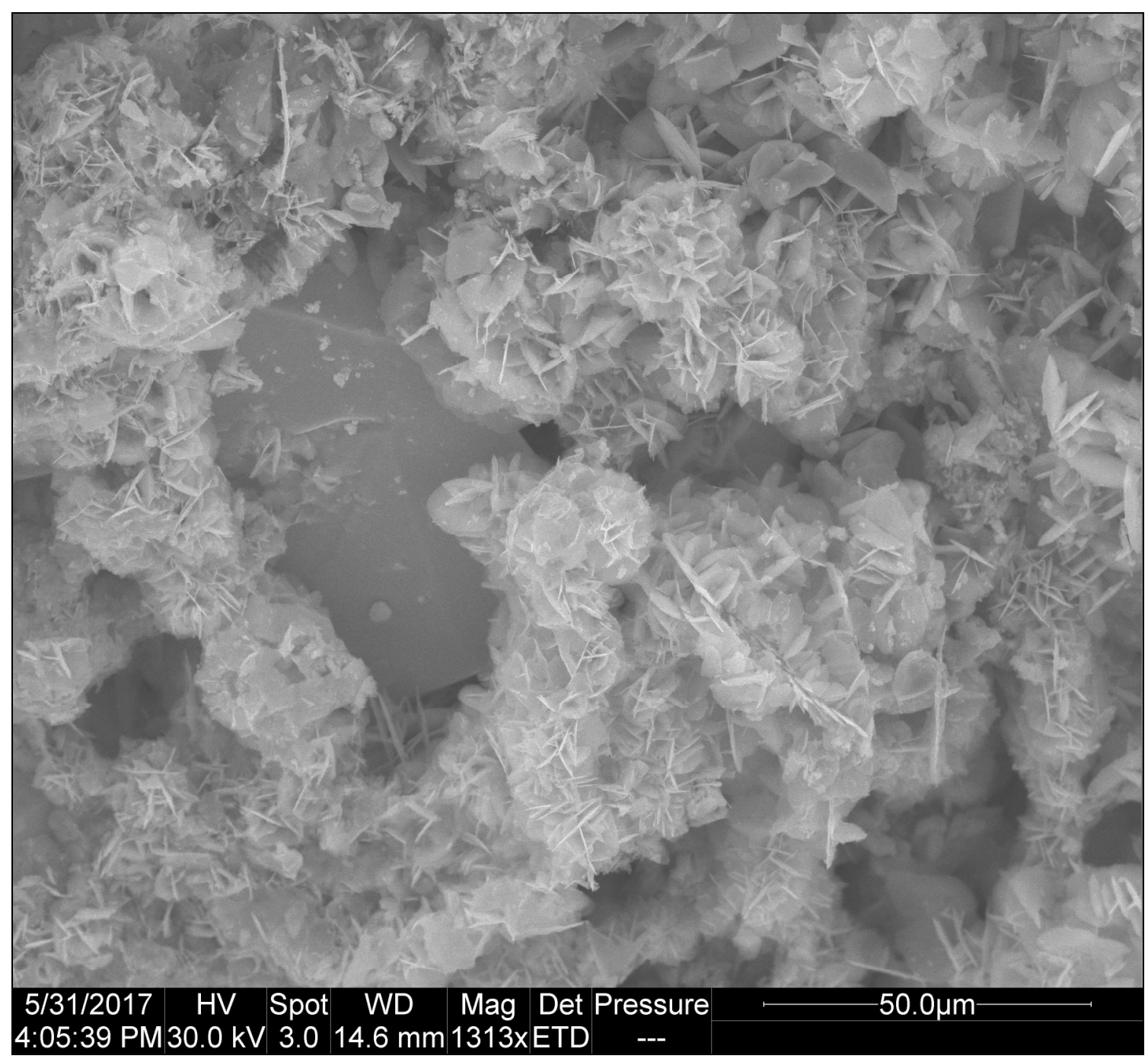

Fig. 13 : Covellite sur galène, Erlenbach. Échantillon ERL 06. Photographie au microscope électronique à balayage, mode électrons secondaires.

\subsection{Dundasite, $\mathrm{PbAl}_{2}\left(\mathrm{CO}_{3}\right)_{2}(\mathrm{OH})_{4} \cdot \mathrm{H}_{2} \mathrm{O}$}

Comme au Rocheux (Theux) ou à Rabotrath (Blondieau et al., 2016), la dundasite se présente

à Erlenbach en petites touffes blanches cotonneuses (Figure 14). Elle n'y est pas vraiment fréquente et se rencontre souvent associée à l'allophane. Son identification est confirmée par diffraction des rayons X. La dundasite, observée également à Poppelsberg, est une espèce discrète mais qui semble faire partie des minéraux observés dans les zones d'altération des minerais sulfurés régionaux. 


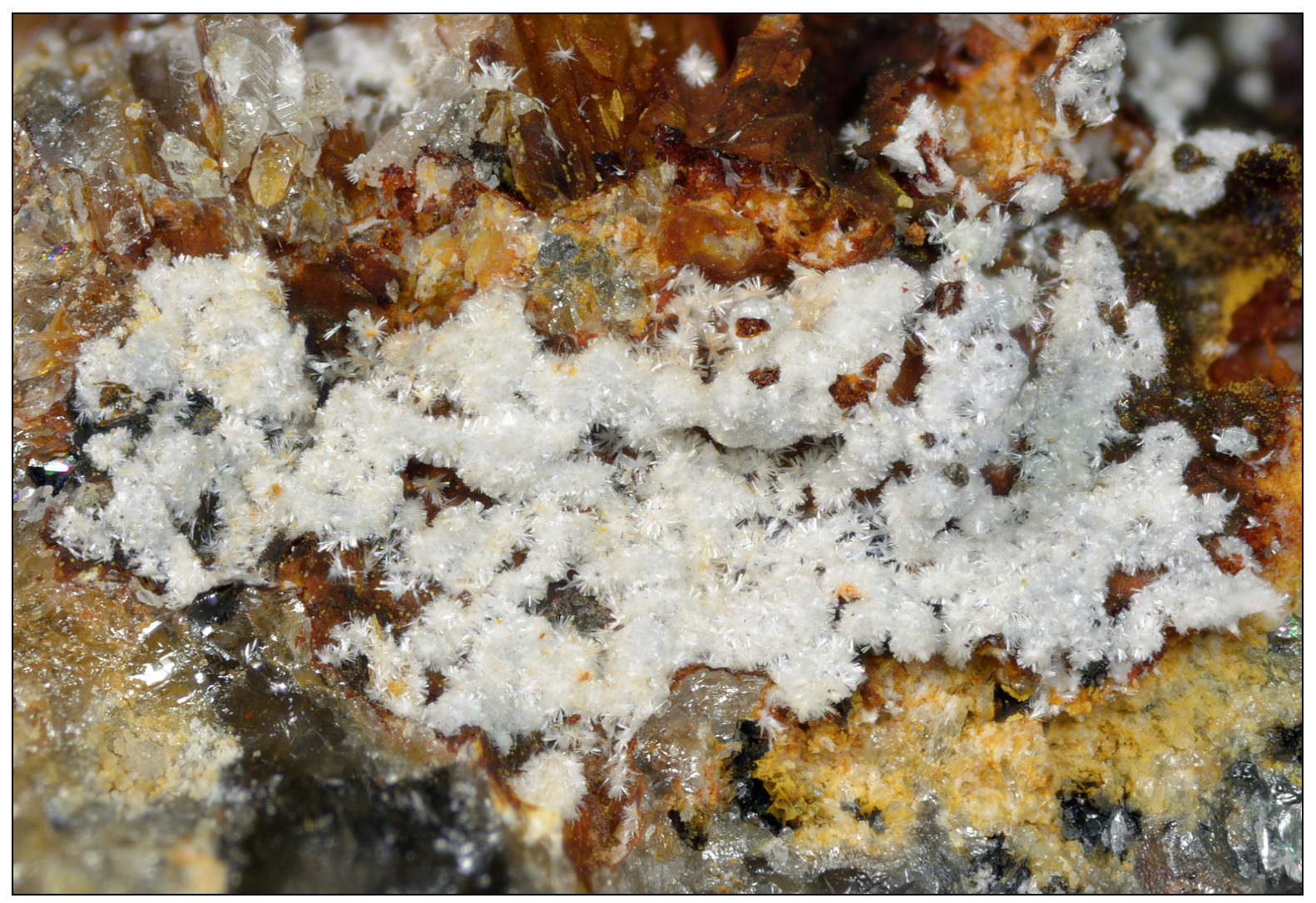

Fig. 14 : Dundasite d'Erlenbach. L = 4,4 mm. (Photo P. Chollet).

\subsection{Galène, $\mathrm{PbS}$}

La galène, identifiée visuellement, se trouve très souvent dans l'argile rouge collante, en rognons corrodés de toute taille, pouvant atteindre exceptionnellement $15 \mathrm{~cm}$ de diamètre. À leur surface, on observe de minuscules cristaux gris de cérusite ou parfois une croûte blanche constituée majoritairement d'anglésite (Figure 7).

Assez rarement, on peut observer des cristaux isolés de galène présentant un octaèdre très allongé. Toujours corrodés, ils peuvent atteindre une longueur de $5 \mathrm{~cm}$ (Figure 15). La galène en cristaux aigus n'est pas fréquente; elle est notamment signalée à Planioles, Lot, France (Lebocey, 2009). Normalement, la galène cubique ne peut présenter des cristaux de cette forme, qui se rapproche du quadroctaèdre caractéristique du système cristallin tétragonal. Toutefois, la morphologie particulière des échantillons d'Erlenbach peut s'expliquer par des phénomènes de croissance, où une direction préférentielle aurait été favorisée au cours de la cristallisation, produisant ainsi des cristaux allongés aux angles inter-faciaux plus aigus que ceux de l'octaèdre. 


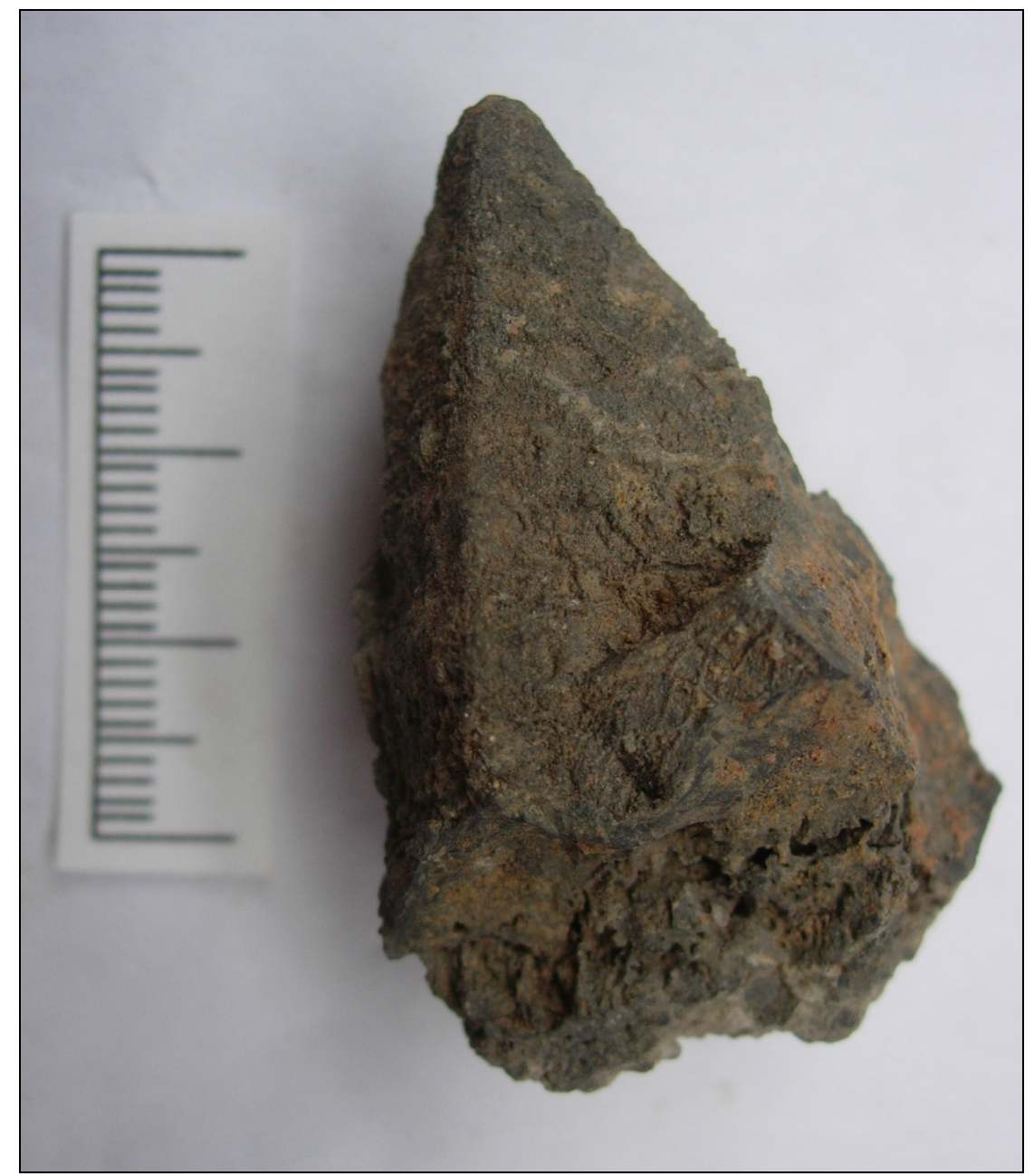

Fig. 15 : Cristal de galène d'Erlenbach montrant un habitus très allongé, (Coll. et Photo : $\mathrm{M}$. Blondieau).

\subsection{Goethite, $\mathrm{FeO}(\mathrm{OH})$}

La goethite mal cristallisée est fréquente à Erlenbach, où elle se présente en grosses masses brunes, parfois pulvérulentes de "limonite". L'analyse par diffraction des rayons X de cette limonite met en évidence son association fréquente à la cérusite et, plus rarement à la plumbojarosite. La goethite se rencontre également en cristaux bruns rhomboédriques (Figure 16) ; il s'agit vraisemblablement d'une pseudomorphose d'un carbonate qu'il n'est plus possible d'identifier.

\subsection{Hématite, $\mathrm{Fe}_{2} \mathrm{O}_{3}$}

L'hématite est reconnue par diffraction des rayons $\mathrm{X}$ en plages d'aspect terreux et de couleur rouge brique. Elle est associée à la goethite dans des blocs riches en limonite. 


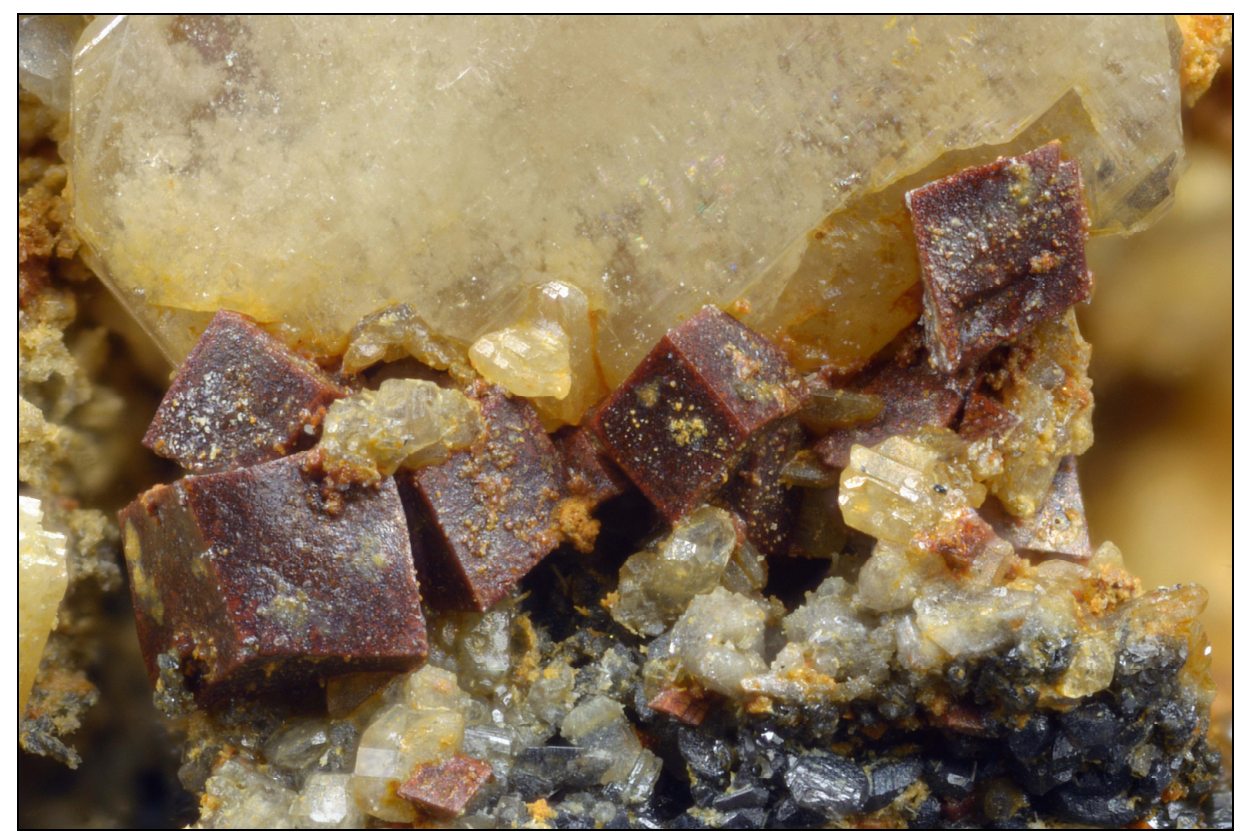

Fig. 16 : Cérusite et cristaux rhomboédriques bruns transformés en goethite. $\mathrm{L}=2,35 \mathrm{~mm}$. (Photo P. Chollet).

\subsection{Hydrohétaerolite, $\mathrm{HZnMn}^{3+}{ }_{1.7} \mathrm{O}_{4}$ et Hétaerolite, $\mathrm{ZnMn}_{2}{ }_{2} \mathrm{O}_{4}$}

Des petites boules noires de quelques millimètres de diamètre sont observées en association avec la smithsonite. Sur cassure fraîche, le minéral est gris acier et très compact; il ne se raie pas à l'ongle contrairement à la plupart des autres oxydes de manganèse observés sur le site (chalcophanite et oxydes de manganèse amorphes). Parfois, on a pu l'observer en petits cristaux (Figure 17).

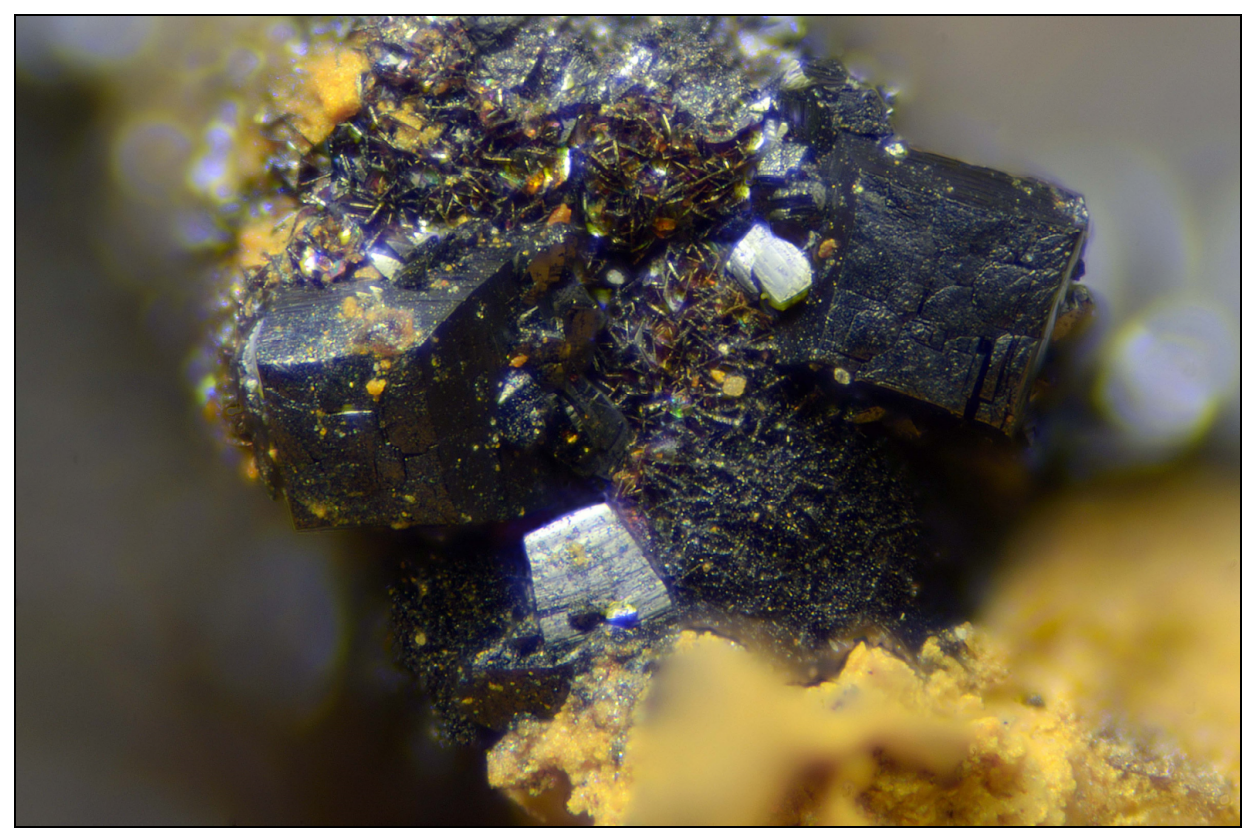

Fig. 17 : Cristaux d'hydrohétaerolite sur chalcophanite d'Erlenbach, $L=0,65 \mathrm{~mm}$. (Photo P. Chollet). 
L'analyse par diffraction des rayons X d'une petite boule noire indique qu'on est en présence d'un minéral du groupe de l'hydrohétaerolite, ce que confirme l'analyse chimique qualitative EDS qui montre la présence des éléments majoritaires $\mathrm{Zn}, \mathrm{Mn}$ et $\mathrm{O}$. Les raies de diffraction de ce minéral d'Erlenbach, comparées à celles de l'hydrohétaerolite de Leadville (Colorado, USA) et d'une hétaerolite de synthèse (Tableau 1) montrent une excellente corrélation avec l'hydrohétaerolite, mais deux raies [à $2,700 \AA$ et à $1,787 \AA]$ pourraient néanmoins être attribuées à l'hétaerolite. Ceci pourrait indiquer l'existence d'une association des deux espèces dans les globules d'Erlenbach. Il en est de même pour un autre échantillon régional provenant de la mine de Schmalgraf, et qui fournit des raies fort comparables. (Tableau 1)

Tableau 1 : Raies de diffraction de l'hydrohetaerolite d'Erlenbach et de celle de Schmalgraf, comparées à celles de l'hydrohetaerolite de Leadville (Colorado, USA) et de l'hetaerolite de synthèse. Les raies mises en rouge pourraient correspondre à l'hetaerolite.

\begin{tabular}{|c|c|c|c|}
\hline $\begin{array}{l}\text { Hydrohétaerolite } \\
\text { (Erlenbach) }\end{array}$ & $\begin{array}{l}\text { Hydrohétaerolite } \\
\text { (Schmalgraf) }\end{array}$ & $\begin{array}{l}\text { Hydrohétaerolite } \\
\text { (Leadville, USA) } \\
\text { Fiche 9-459 }\end{array}$ & $\begin{array}{c}\text { Hétaerolite } \\
\text { (Syn) } \\
\text { Fiche } 24-1133\end{array}$ \\
\hline $\mathrm{d}(\AA)$ & $\mathrm{d}(\AA)$ & $\mathrm{d}(\AA)$ & $\mathrm{d}(\AA)$ \\
\hline & $\begin{array}{c}4,85(2) \\
3,032(46)\end{array}$ & & $4,87(10)$ \\
\hline 3,020 (29) & $3,023(41)$ & $3,02(70)$ & 3,047 (45) \\
\hline $2,868(21)$ & $2,865(31)$ & $2,87(40)$ & $2,862(19)$ \\
\hline $2,700(27)$ & $2,698(31)$ & & $2,715(65)$ \\
\hline $2,667(22)$ & $2,681(42)$ & $2,66(80)$ & \\
\hline $2,467(100)$ & $2,469(100)$ & $2,47(100)$ & $\begin{array}{c}\mathbf{2 , 4 6 6 ( 1 0 0 )} \\
2,432(12) \\
2,311(10)\end{array}$ \\
\hline $\begin{array}{l}2,265(5) \\
2,222(5)\end{array}$ & $2,279(3)$ & $2,25(10)$ & \\
\hline & & $2,13(10)$ & \\
\hline $2,024(9)$ & $2,026(11)$ & $2,03(20)$ & $2,022(16)$ \\
\hline 1,787 (4) & $1,782(4)$ & & $1,798(7)$ \\
\hline $1,778(7)$ & & $1,773(10)$ & \\
\hline & $1,747(4)$ & & $1,760(15)$ \\
\hline $1,730(4)$ & $1,732(5)$ & $1,723(20)$ & \\
\hline $1,683(13)$ & $1,684(13)$ & $1,686(20)$ & $\begin{array}{c}1,684(12) \\
1,621(9)\end{array}$ \\
\hline $1,617(5)$ & $1,618(6)$ & $1,613(10)$ & \\
\hline
\end{tabular}

Kolitsch et al. (2014) ont signalé à l'Esperanza mine (Laurion, Grèce) la présence de cristaux constitués d'un cœur d'hétaerolite avec une enveloppe d'hydrohétaerolite. Ces deux espèces proches peuvent donc être associées et cela semble être bien le cas à Erlenbach mais aussi à 
Schmagraf. Les échantillons que nous avons étudiés semblent montrer une prédominance de l'hydrohetaerolite mais un échantillon de Moresnet étudié par Fransolet \& Mélon (1975) a été identifié comme une hetearolite. Ces auteurs ont cependant précisé qu'une confusion avec l'hydrohétaerolite était possible. Il semble donc que des associations de ces deux espèces existent bien à Erlenbach, Schmalgraf et Moresnet mais avec des proportions pouvant varier d'un échantillon à l'autre.

\subsection{Hydrozincite, $\mathrm{Zn}_{5}\left(\mathrm{CO}_{3}\right)_{2}(\mathrm{OH})_{6}$}

L'identification de l'hydrozincite est réalisée sur base de son radiogramme de poudre de rayons X. On l'a observée fort rarement en croûtes blanches d'un ou deux centimètres d'épaisseur et d'aspect crayeux, associées à de petites plages de smithsonite blanche sur smithsonite grise (Figure 18). Le minéral observé sous une lampe ultra-violette émettant des ondes courtes $(254 \mathrm{~nm})$ manifeste une fluorescence blanc bleuté très vive et très caractéristique de l'espèce. La smithsonite blanche d'Erlenbach pourrait parfois être confondue avec de l'hydrozincite.

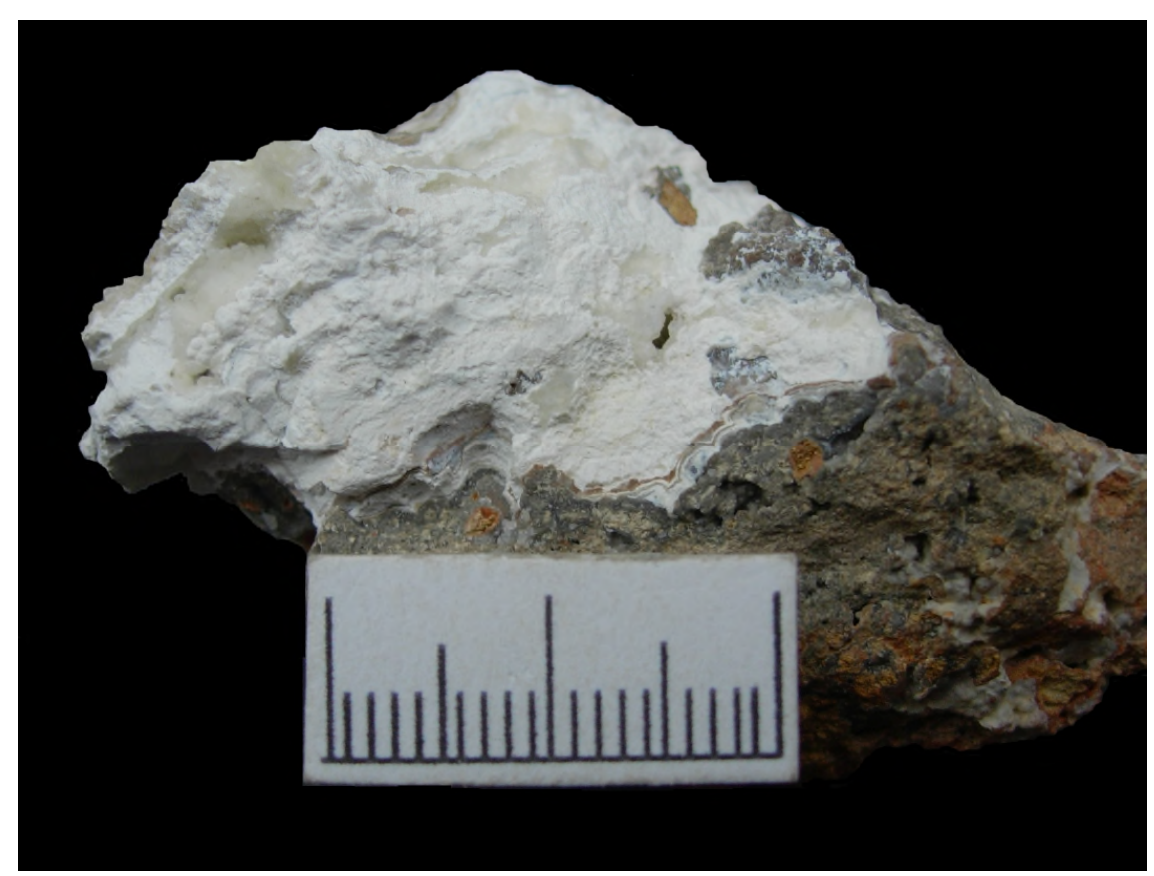

Fig. 18 : Hydrozincite sur smithsonite d'Erlenbach. (Coll. et Photo : M. Blondieau).

\subsection{Kaolinite, $\mathrm{Al}_{2} \mathrm{Si}_{2} \mathrm{O}_{5}(\mathrm{OH})_{4}$}

L'analyse par diffraction des rayons $\mathrm{X}$ d'un minéral blanc argileux sur goethite, et visuellement déterminé comme de l'halloysite, montre qu'il s'agit de kaolinite. L'halloysite, bien que soupçonnée, n'a pas été confirmée à Erlenbach. 


\subsection{Malachite, $\mathrm{Cu}_{2} \mathrm{CO}_{3}(\mathrm{OH})_{2}$}

Une petite pustule verte sur un gros rognon de galène corrodée en cérusite et anglésite est visuellement déterminée comme de la malachite. Bien que peu abondant dans la région, le cuivre accompagne parfois la blende zonée comme cela a déjà été observé sur certains échantillons de Schmalgraf.

\subsection{Marcasite, $\mathrm{FeS}_{2}$}

La marcasite est parfois observée avec galène dans les très rares échantillons de « schalenblende» (sphalérite rubanée) récoltés sur le site. L’identification de l'espèce est confirmée par diffraction des rayons X. La marcasite fait partie de l'assemblage classique des minerais $\mathrm{Pb}-\mathrm{Zn}$ sulfurés régionaux.

\subsection{Minium, $\mathrm{Pb}^{2+} \mathrm{Pb}^{4+} \mathrm{O}_{4}$}

Des petites plages d'un minéral pulvérulent rouge ont été observées à l'intérieur d'un gros nodule de galène presque complètement transformé en anglésite et cérusite (Figure 19). Visuellement déterminé comme minium, l'analyse par diffraction des rayons $\mathrm{X}$ de ce minéral confirme l'identification de l'espèce. Les photos en microscopie électronique (Figure 20) montrent sous fort grossissement le minium en globules cristallins d'à peine un micron, déposés sur des cristaux de cérusite. Des analyses chimiques qualitatives par EDS indiquent la présence majoritaire de $\mathrm{Pb}$ et $\mathrm{O}$.

Le minium n'est pas une espèce reprise dans le livre "Les minéraux de Belgique" (Hatert et al., 2002). Ce minéral ne doit cependant pas être vraiment rare en Belgique car nous l'avons également identifié par diffraction des rayons X à Ave-et-Auffe, avec galène et barite (bois du Roptai), à la carrière Lhoist de Rochefort, avec smithsonite, et dans le bois de Vodecée à Sautour, également sur smithsonite. 
Bulletin de la Société des Sciences de Liège, Vol. 87, articles, 2018, p. 22 - 55

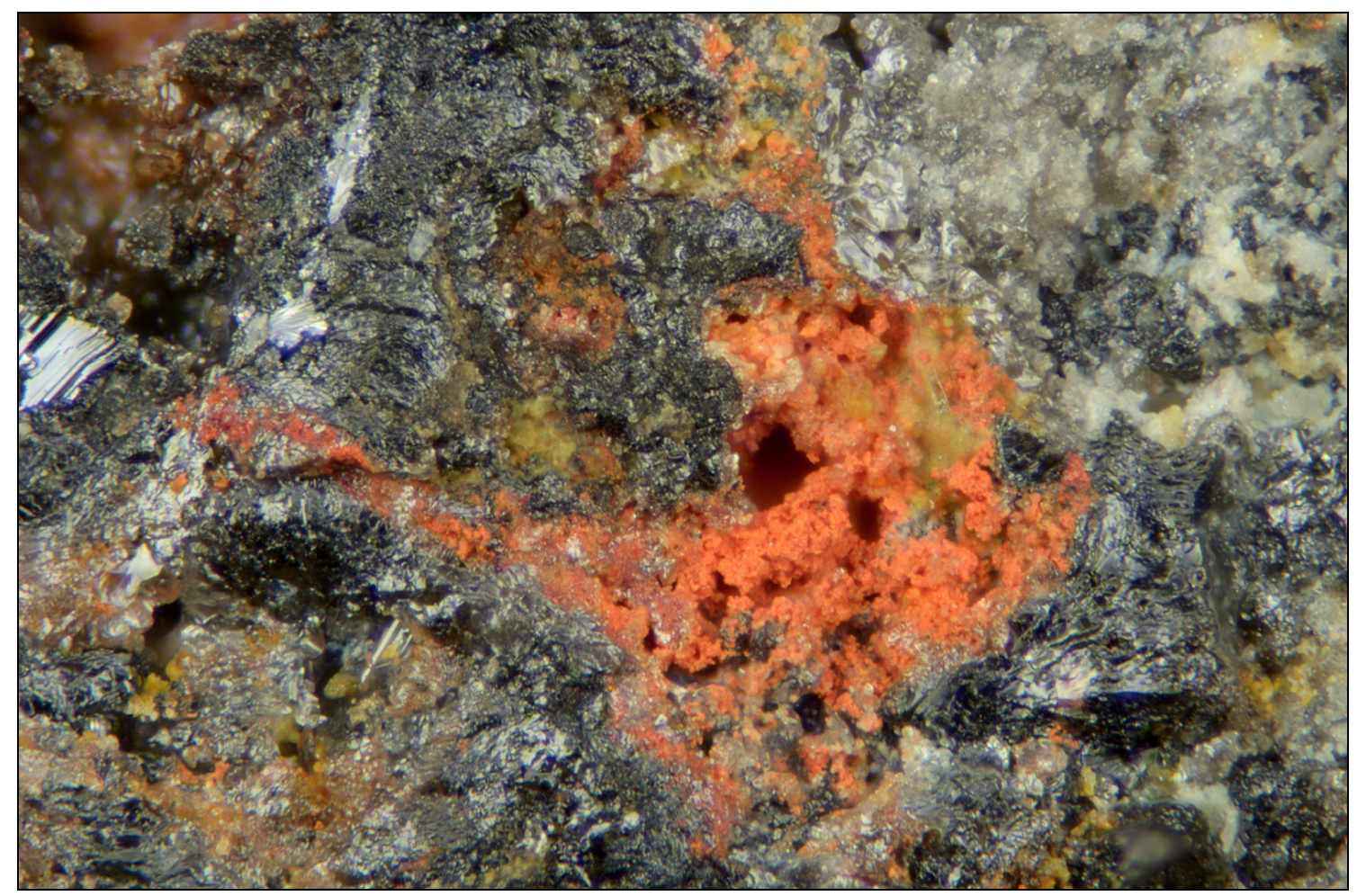

Fig. 19: Minium avec cérusite, Erlenbach. $\mathrm{L}=0,85 \mathrm{~mm}$. (Photo P. Chollet).
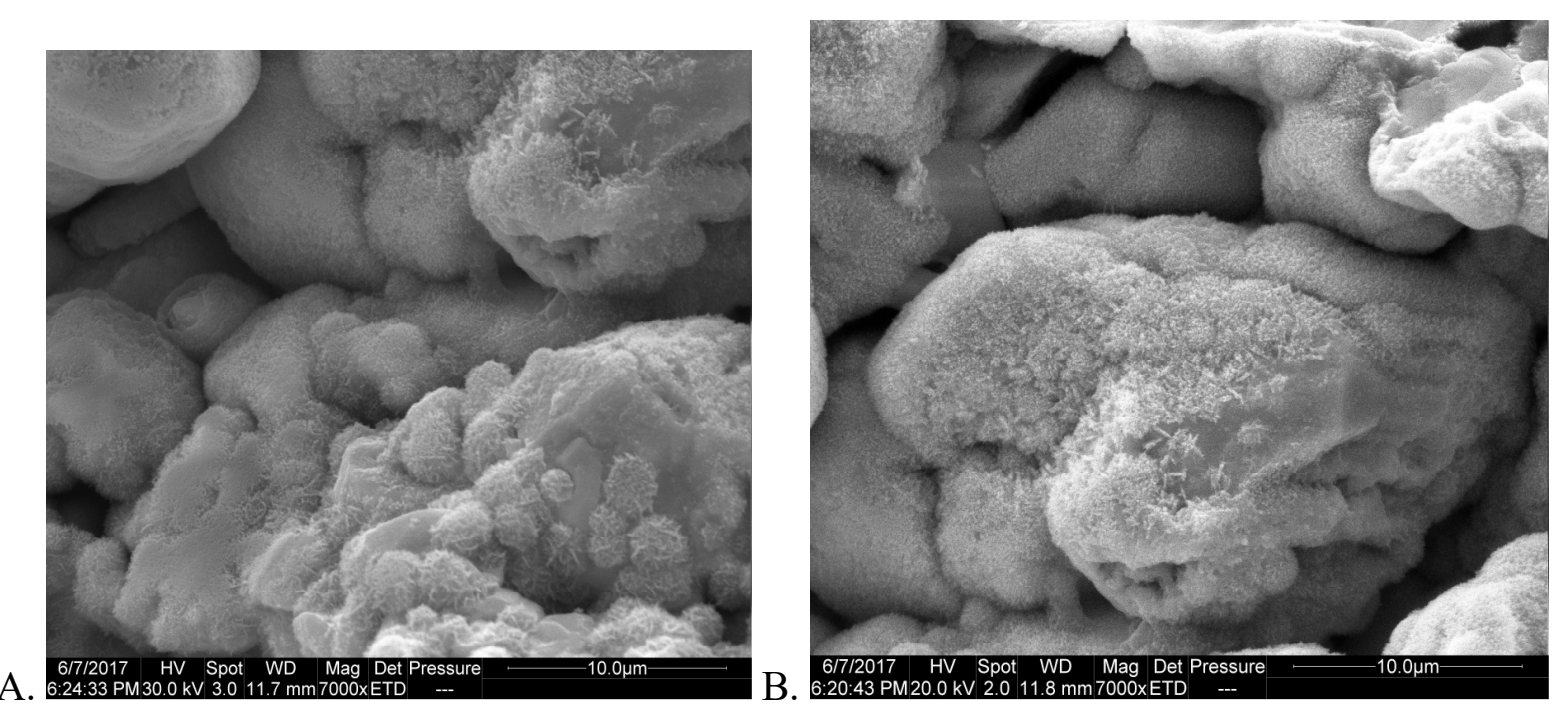


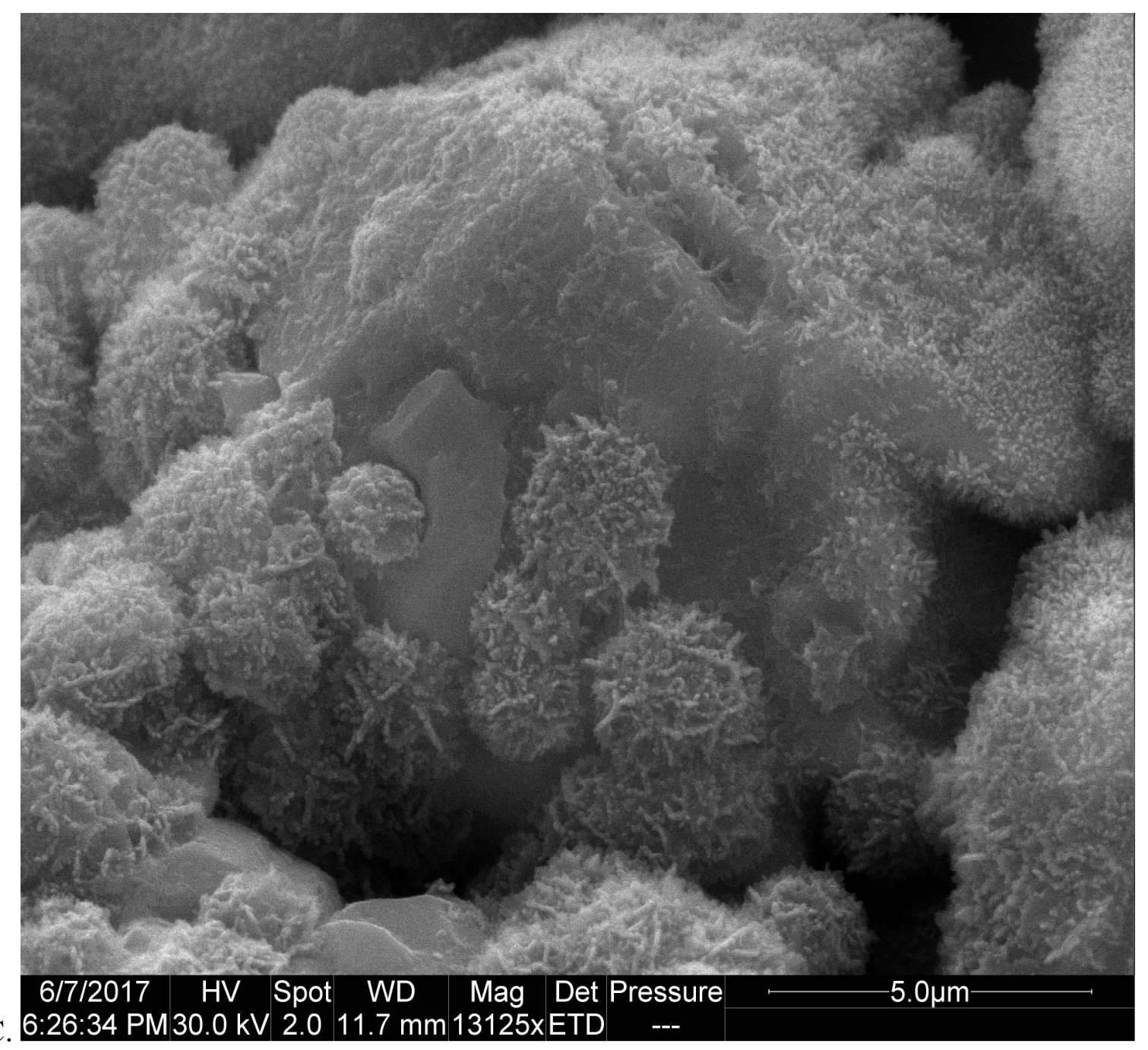

Fig. 20 : Échantillon ERL 04. Photographies au microscope électronique à balayage, mode électrons secondaires. Minium en petits globules cristallins sur cérusite, Erlenbach.

\subsection{Plumbojarosite, $\mathrm{Pb}_{0,5} \mathrm{Fe}_{3}\left(\mathrm{SO}_{4}\right)_{2}(\mathrm{OH})_{6}$}

L'analyse par diffraction des rayons X d'un minéral d'aspect argileux, se présentant en petites paillettes brunes chatoyantes, montre qu'il s'agit d'un membre du groupe de la jarosite. Son association avec la galène permet de soupçonner la plumbojarosite, ce qu'un examen attentif des raies de diffraction semble confirmer. Des analyses chimiques qualitatives par EDS indiquent bien la présence des éléments $\mathrm{Fe}, \mathrm{Pb}, \mathrm{S}$ et $\mathrm{O}$, confirmant ainsi l'espèce. Les photographies en microscopie électronique montrent que ces plages sont constituées de l'association de nombreux petits cristaux très bien formés, d'une taille de quelques microns seulement (Figure 21). 

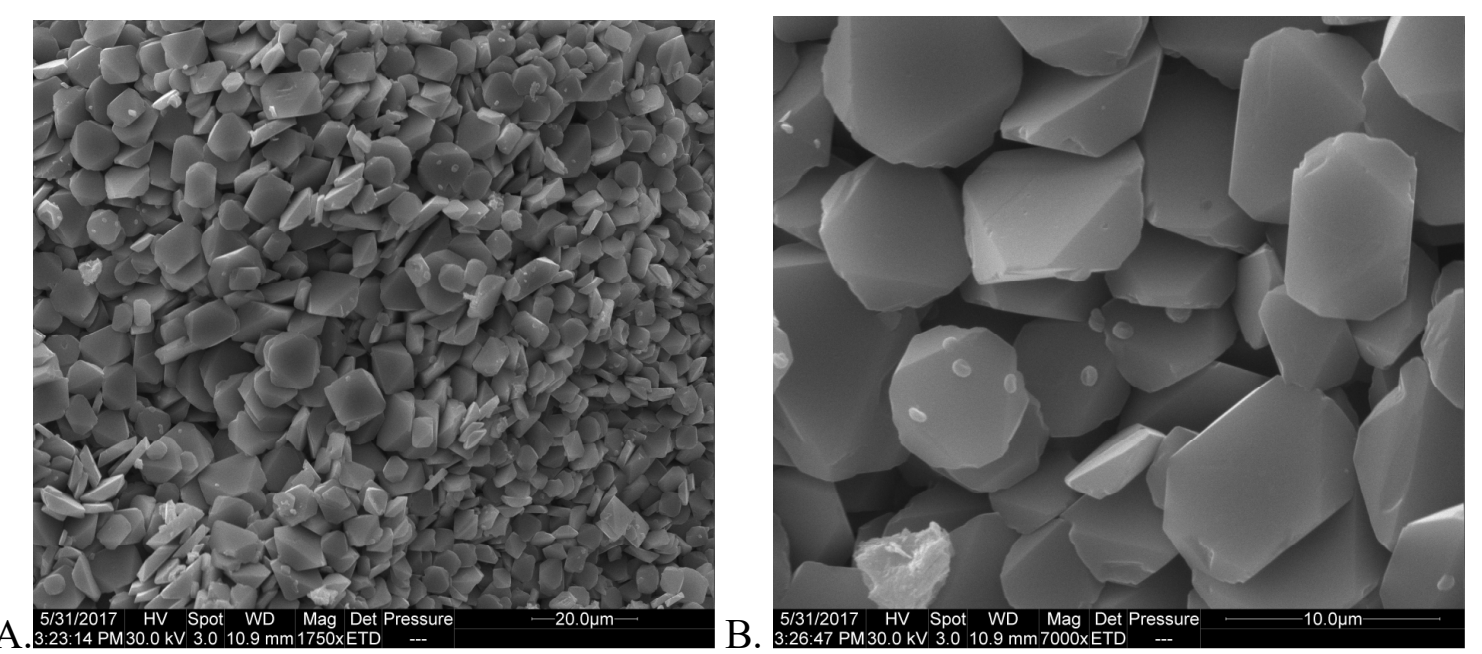

Fig. 21 : Plumbojarosite d'Erlenbach. Échantillon ERL 05. Photographies au microscope électronique à balayage, mode électrons secondaires.

\subsection{Pyromorphite, $\mathrm{Pb}_{5}\left(\mathrm{PO}_{4}\right)_{3} \mathrm{Cl}$}

De petits globules verts, identifiés visuellement comme étant de la pyromorphite, ont été confirmés par diffraction des rayons X. Les analyses chimiques qualitatives par EDS mettent en évidence les éléments majoritaires $\mathrm{Pb}, \mathrm{P}$ et $\mathrm{O}$ ainsi que $\mathrm{Fe}, \mathrm{Ca}, \mathrm{Sr}$ et $\mathrm{Al}$ fort minoritaires. Le chlore n'a pas été détecté, mais il s'agit sans doute d'un problème de sensibilité. En microscopie électronique, ces cristaux apparaissent fort corrodés (Figure 22).

Des analyses chimiques qualitatives d'une autre population de cristaux, visuellement de couleur brune, indiquent une composition chimique proche de celle des cristaux verts et confirme qu'il s'agit également de pyromorphite. En microscopie électronique, ces cristaux qui ne dépassent pas 100 microns se présentent en beaux barillets hexagonaux, avec les formes $\{10.0\},\{10.1\}$ et $\{00.1\}$ (Figure 23). La pyromorphite n'est pas une espèce fréquente à Erlenbach. 


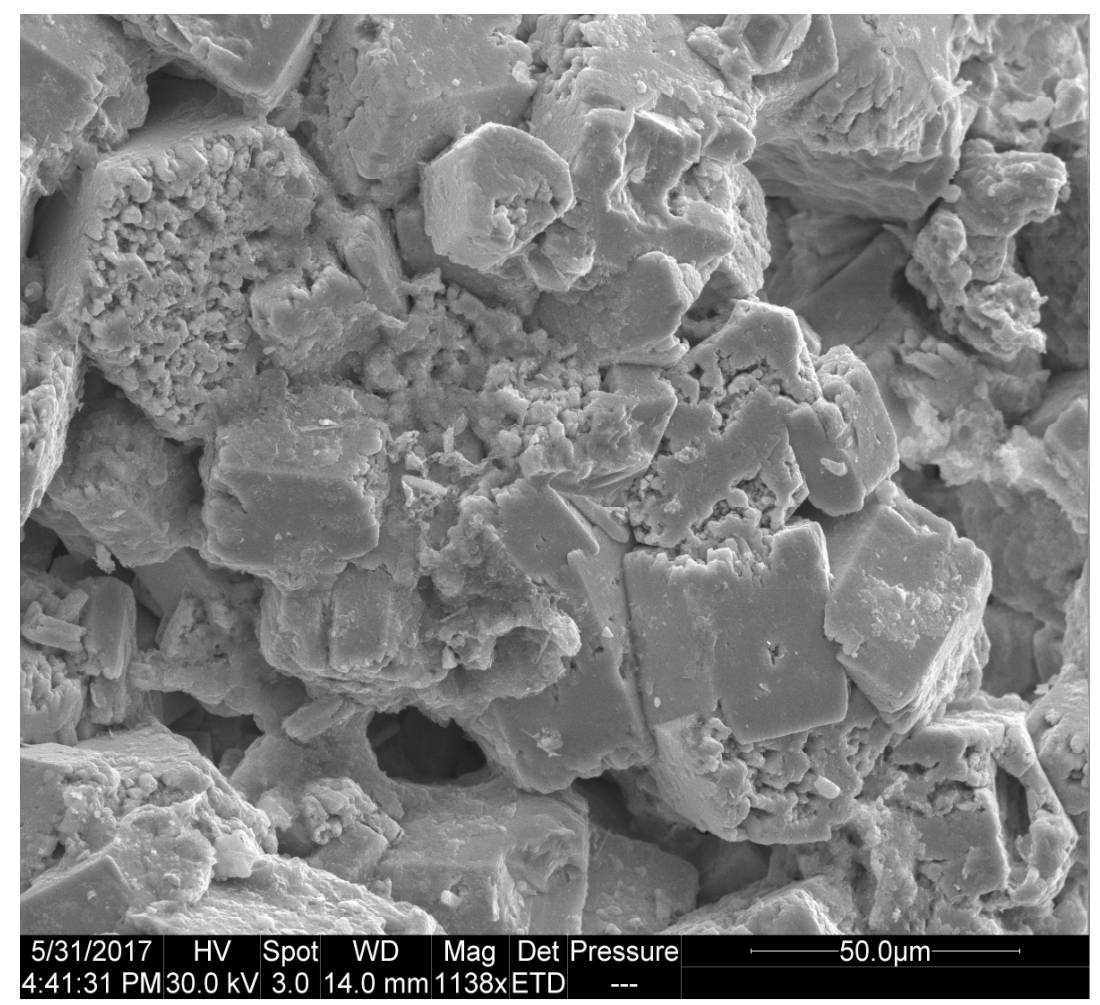

Fig. 22 : Cristaux de pyromorphite verte montrant des figures de corrosion, Erlenbach. Échantillon ERL 09. Photographie au microscope électronique à balayage, mode électrons secondaires
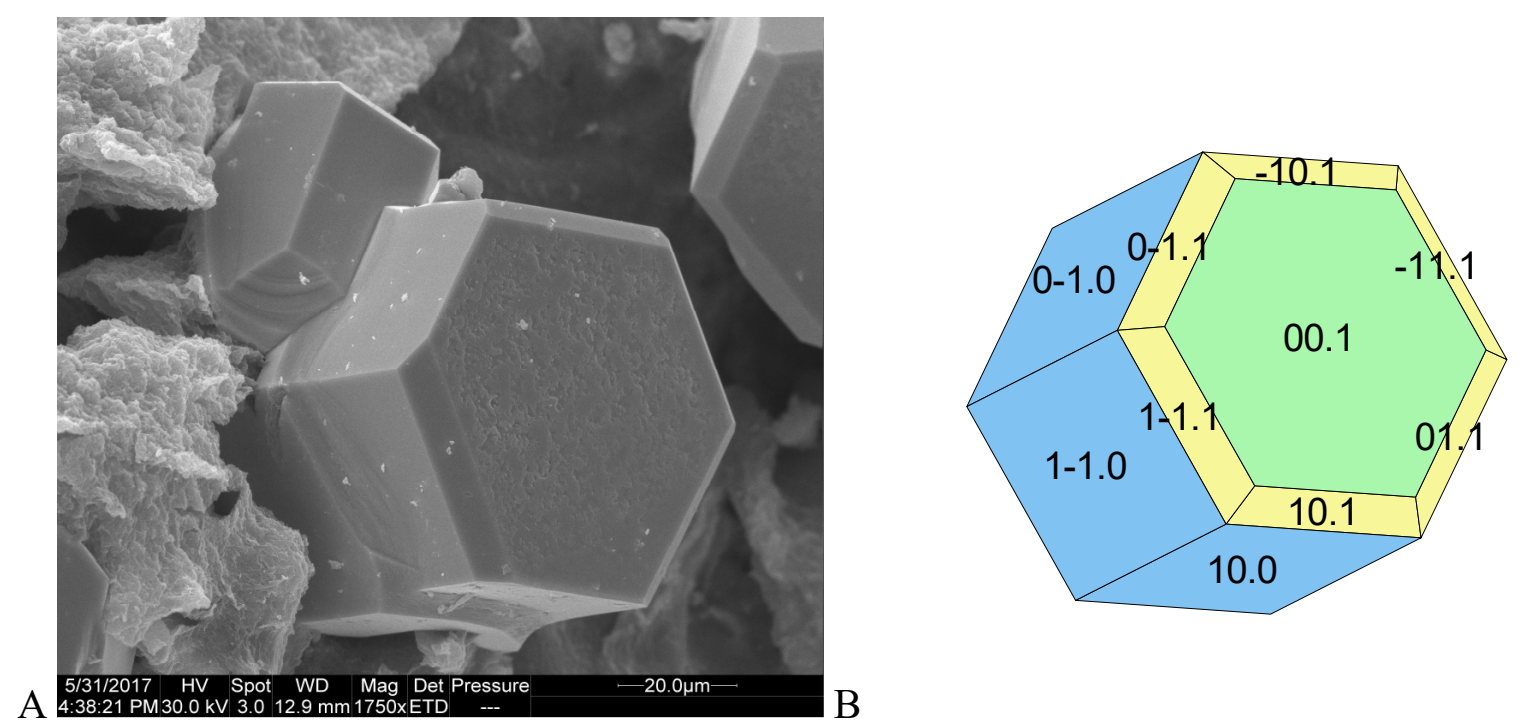

Fig. 23 : Cristaux de pyromorphite brune, Erlenbach. Échantillon ERL 08. A. Photographie au microscope électronique à balayage, mode électrons secondaires. B. Morphologie des cristaux (Favreau, 2006).

\subsection{Quartz, $\mathrm{SiO}_{2}$}

Des fragments de quartz, souvent millimétriques à centimétriques, sont observés nombreux comme éléments d'une brèche rouge cimentée par de la cérusite. Des cristaux de quartz, 
parfois centimétriques et parfois aussi légèrement enfumés, peuvent encore être ramassés sur les haldes après les pluies (Figure 24). Dans la région, le quartz enfumé a été également observé sur le site de Schimper (filon de Bleiberg) (Blondieau \& Polrot, 2011).

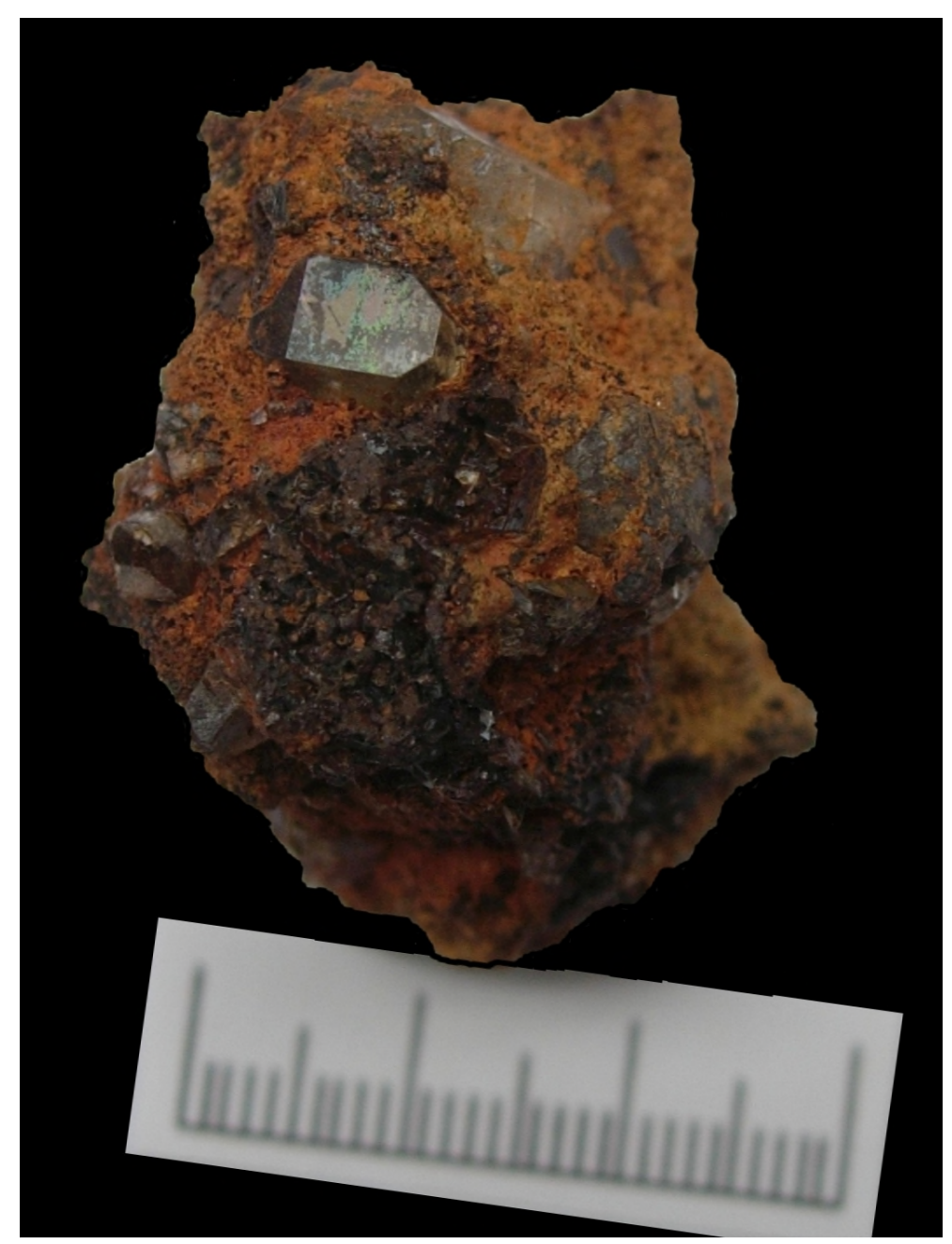

Fig. 24 : Cristal de quartz centimétrique dans brèche limoniteuse et siliceuse d'Erlenbach. (Coll. et Photo : M. Blondieau).

\subsection{Smithsonite, $\mathrm{ZnCO}_{3}$}

La smithsonite, dans sa variété "calamine roche », est très abondante dans l'argile rouge et peut se trouver en blocs parfois de grande taille. On la reconnaît aisément à sa couleur gris blanchâtre et son aspect mat généralement scoriacé, que certains appellent "dry bone habitus". Effectivement, la majorité des échantillons sont fort peu esthétiques et ressemblent assez à des bouts de vieux os blanchis au soleil (Figure 25). 

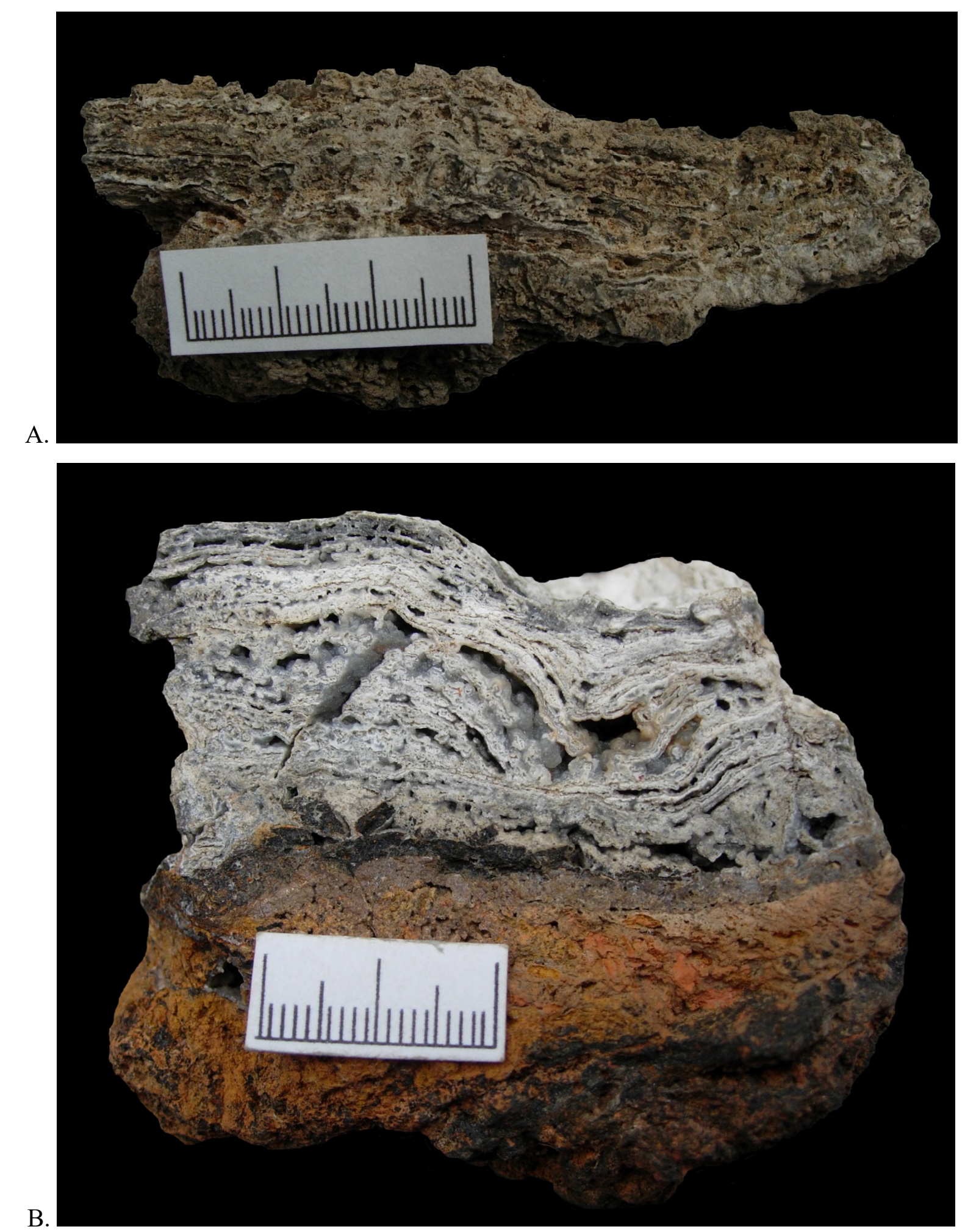

Fig. 25 : «Calamine » (smithsonite) d'Erlenbach. (Coll. et Photos : M. Blondieau).

Les échantillons en petits mamelons caractéristiques de l'espèce sont fort rares et de couleur souvent terne, variant le plus souvent du blanc au gris parfois presque noir. La smithsonite de couleur blanche n'est pas fréquente à Erlenbach (Figure 26). 
Bulletin de la Société des Sciences de Liège, Vol. 87, articles, 2018, p. 22 - 55
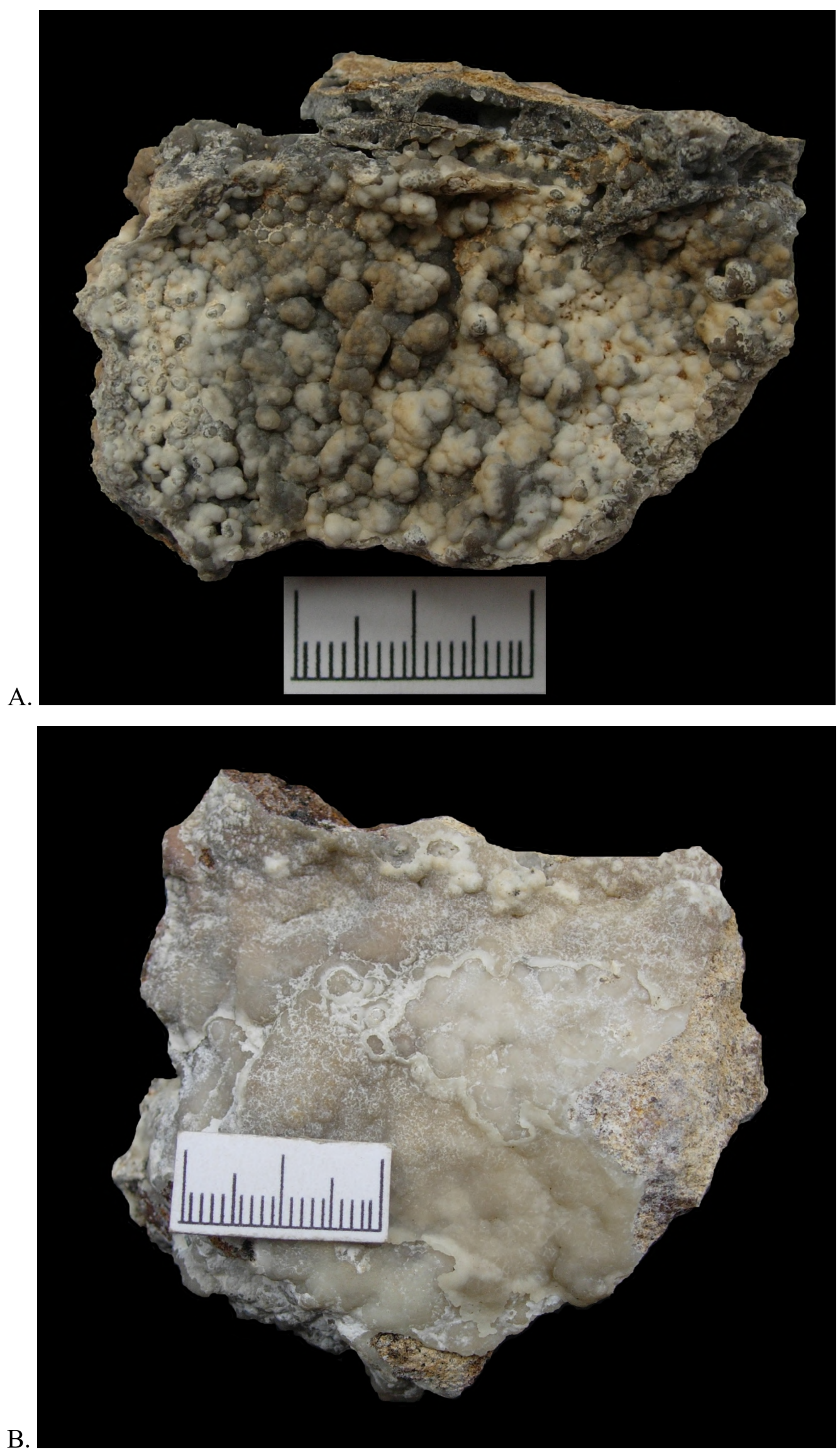

Fig. 26 : Smithsonite mamelonnée d'Erlenbach. (Coll. et Photos : M. Blondieau). 
Un élément de la brèche brune, visuellement identifiée comme de la sidérite fort altérée, a montré par diffraction des rayons $\mathrm{X}$ qu'il était constitué d'un mélange de smithsonite et de goethite. Sur ce diffractogramme, on voit des pics très étroits pour la smithsonite et des pics fortement évasés pour la goethite. La smithsonite est donc bien cristalline, alors que la goethite apparaît assez mal cristallisée. Ces éléments bruns de la brèche doivent donc être considérés comme une calamine pauvre très ferrugineuse. À la surface de ces blocs, on observe fréquemment des pellicules grises d'épaisseur millimétrique constituées de nombreux petits cristaux de smithsonite, parfois arrondis en "œufs de fourmis".

\subsection{Soufre, $S$}

Des petits cristaux jaunes translucides sont observés dans des fissures de la blende zonée. A Rabotrath, des cristaux comparables ont été identifiés comme soufre (Blondieau et al., 2016). L'analyse par diffraction des rayons $\mathrm{X}$ de ces petits cristaux d'Erlenbach confirme l'identification. Des croûtes blanc-jaunâtre de quelques millimètres d'épaisseur sont également observées sur un échantillon de blende zonée; il s'agit également de soufre, produit d'altération des sulfures fréquemment observé dans les anciennes haldes soumises aux altérations météoriques.

\subsection{Sphalérite, $(\mathrm{Zn}, \mathrm{Fe}) \mathrm{S}$}

Comme dans la plupart des autres sites du district métallifère $\mathrm{Pb}-\mathrm{Zn}$ de l'est de la Belgique, le minerai de zinc sulfuré à Erlenbach se présente sous la forme de «blende zonée », appelée aussi «blende rubanée» ou «schalenblende ». À Erlenbach, elle semble très rare et seuls quelques petits fragments ont pu être trouvés dans le déblai. Une analyse par diffraction des rayons X de ce minerai montre qu'il s'agit, comme à Rabotrath (Blondieau et al., 2016), de sphalérite très pure sans würtzite (Figure 27). 

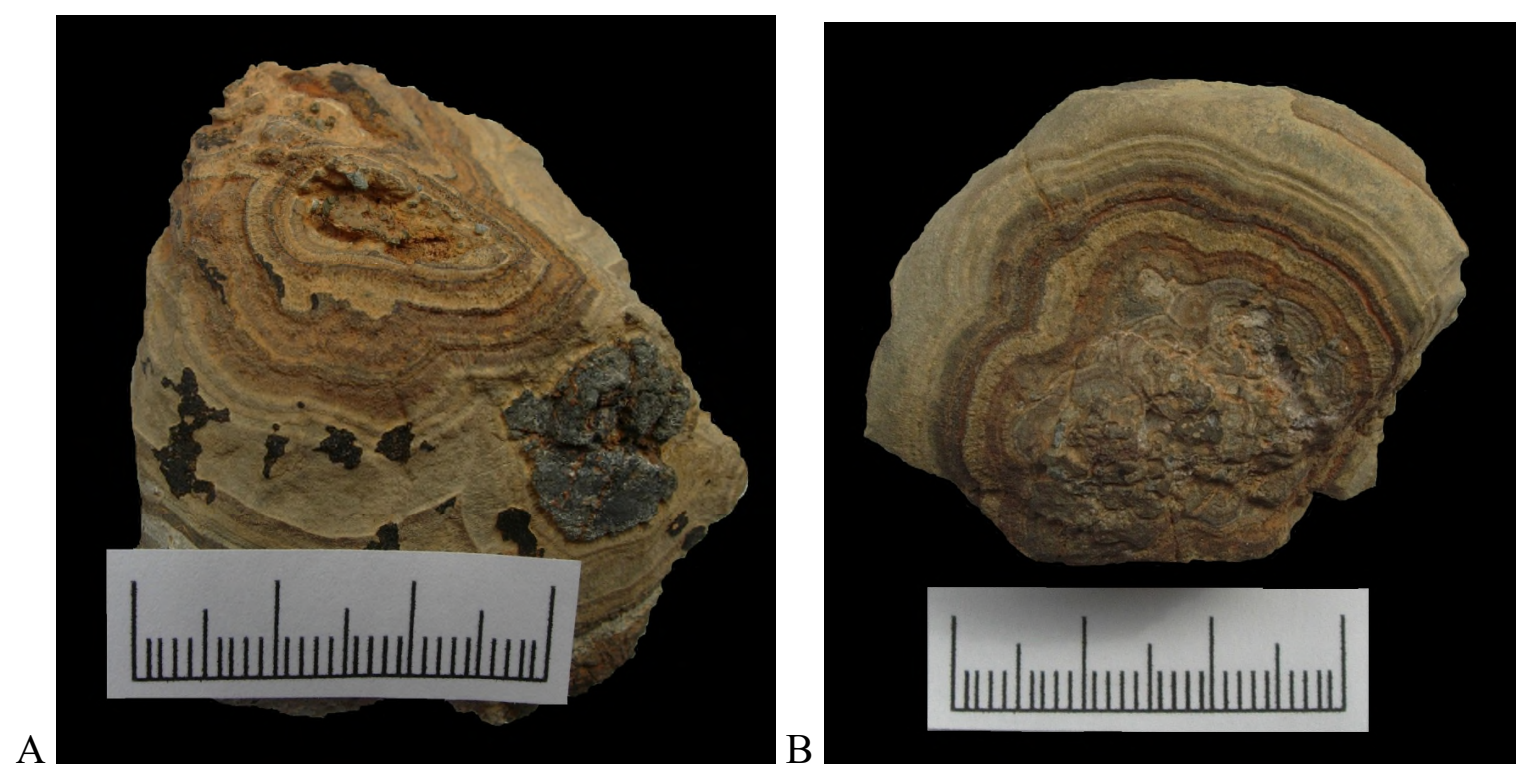

Fig. 27 : Blende zonée ("schalenblende" = sphalérite rubanée) d'Erlenbach avec noyau de galène (partie grise). Recto-verso du même échantillon. (Coll. et Photos : M. Blondieau).

\subsection{Espèce minérale non identifiée, $\mathrm{Al}-\mathrm{Si}-\mathrm{Zn}-\mathrm{O}$}

Le minéral se présente avec allophane et dundasite en petites plages blanches de texture argileuse. Visuellement, il ressemble à de l'halloysite. Des analyses par diffraction des rayons $\mathrm{X}$ en mode Debye-Scherrer ont été réalisées sur ce matériel avec un diffractomètre Philips (échantillon 1) et avec un diffractomètre à 4 cercles Rigaku (échantillons 2 et 3 ). Ces analyses fournissent des valeurs très reproductibles qui ne correspondent apparemment à aucune espèce connue actuellement (Tableau 2). Les analyses chimiques qualitatives EDS réalisées sur de nombreux spots de cette matière indiquent de manière très reproductible la présence des seuls éléments $\mathrm{Al}, \mathrm{Si}, \mathrm{Zn}$ et $\mathrm{O}$. Il s'agit donc d'un alumino-silicate de zinc.

Les images en microscopie électronique à balayage montrent un minéral en feuillets (Figure 28A) qui peuvent s'assembler en petits édifices cristallins d'une dizaine de microns dans de minuscules cavités (Figure 28B). Il est observé sur allophane et est associé à des petits globules de chalcophanite. 
Tableau 2 : Raies de diffraction observées sur le minéral Al-Si-Zn-O non déterminé. L'échantillon 1 a été diffracté avec le diffractomètre de poudre Philips utilisant un tube au fer, les échantillons 2 et 3 ont été diffractés en mode poudre avec un diffractomètre à quatre cercles Rigaku utilisant un tube au molybdène.

\begin{tabular}{ccc}
\hline $\begin{array}{c}\text { Échantillon 1 } \\
\text { Erlenbach) }\end{array}$ & $\begin{array}{c}\text { Échantillon 2 } \\
\text { (Erlenbach) }\end{array}$ & $\begin{array}{c}\text { Échantillon 3 } \\
\text { (Erlenbach) }\end{array}$ \\
$\mathrm{d}(\AA)$ & $\mathrm{d}(\AA)$ & $\mathrm{d}(\AA)$ \\
& & \\
\hline $\mathbf{1 1 , 8 2 7}(\mathbf{9 0})$ & $\mathbf{1 1 , 8 6 ( T F )}$ & $\mathbf{1 1 , 8 6}(\mathbf{T F})$ \\
- & - & 8,83 \\
$9,803(19)$ & - & - \\
$5,938(44)$ & $5,97(\mathrm{~F})$ & $5,92(\mathrm{~F})$ \\
$\mathbf{3 , 9 5 4}(\mathbf{1 0 0})$ & $\mathbf{3 , 9 4}(\mathbf{T F})$ & $\mathbf{3 , 9 4}(\mathbf{T F})$ \\
$2,607(72)$ & 2,61 & 2,62 \\
$2,478(85)$ & 2,49 & 2,48 \\
$2,267(55)$ & 2,28 & 2,27 \\
$2,045(25)$ & 2,04 & 2,04 \\
$1,836(16)$ & 1,84 & 1,84 \\
$1,707(10)$ & 1,70 & 1,70 \\
$1,685(17)$ & 1,63 & 1,63 \\
- & $\mathbf{1 , 5 2}(\mathbf{F})$ & $\mathbf{1 , 5 2}(\mathbf{F})$ \\
- & - & 1,47 \\
- & 1,42 & 1,42 \\
- & 1,31 & 1,31 \\
- & 1,26 & 1,26 \\
- & 1,21 & 1,20 \\
- & 1,07 & 1,07 \\
- & 0,99 & 0,99 \\
- & 0,88 & 0,88 \\
\hline
\end{tabular}




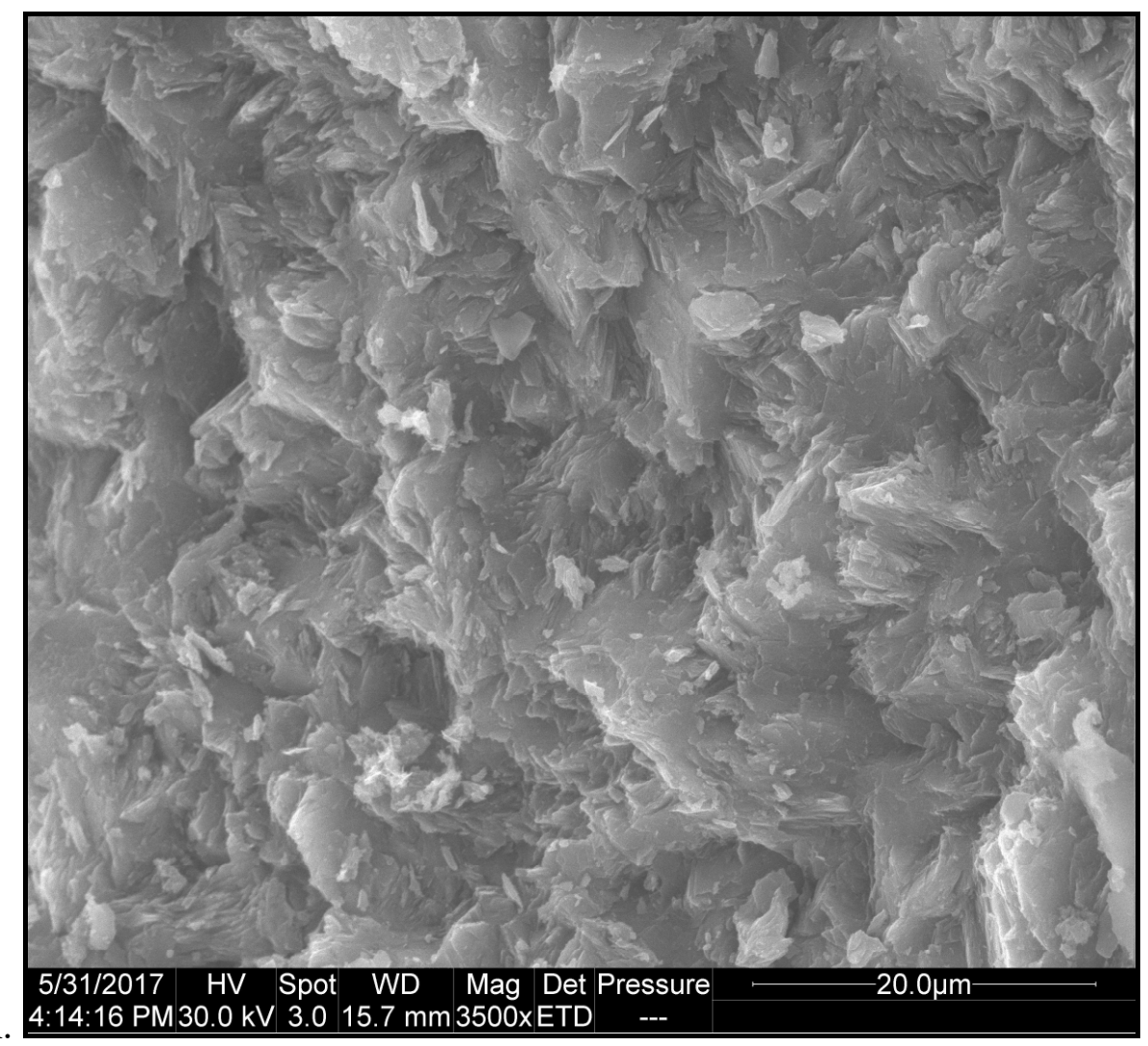

A. 4:14:16 PM30.0 kV $3.015 .7 \mathrm{~mm} 3500 \times \mathrm{xETD}$

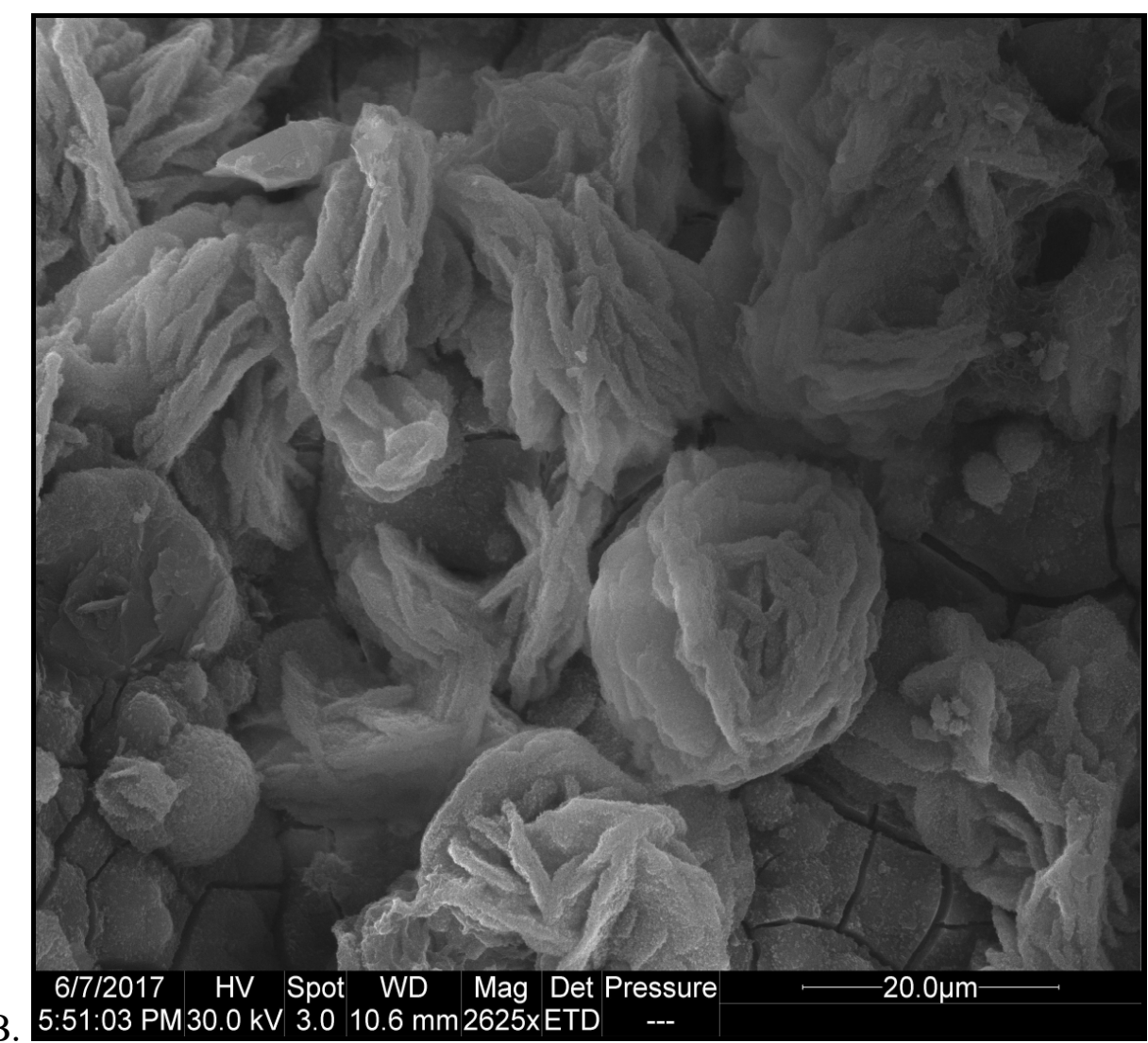

Fig. 28 : Minéral indéterminé Al-Si-Zn-O, échantillon ERL 07. (A) Structure en feuillets. (B) Feuillets assemblés en petites rosettes sur allophane craquelée. Les petites boules sont constituées de chalcophanite. Photographies au microscope électronique à balayage, mode électrons secondaires. 


\section{Conclusions}

L'abondance des argiles rouges collantes et la présence de blocs avec fragments de quartz cimentés par de la cérusite pourraient indiquer que l'essentiel des minéralisations d'Erlenbach se trouvait selon la classification de Dejonghe \& Yans (1983) dans un gisement "lié à l'inconformité paléozoïque-Mésozoïque" (Figure 5). Pour ces auteurs, il s'agit en fait d'amas de contact particuliers provenant du remaniement mécanique à faible distance de gisements préexistants. Ces lentilles, toujours horizontales et sans racines filoniennes, sont essentiellement argileuses avec des teneurs en minerai généralement basses. Comme exemple, ils citent certaines parties du gisement d'Eschbroich mais aussi des indices miniers à Elsenbach pour lesquels ils signalent un volume de 40000 à 50000 tonnes de minerai. Mais s'agit-il vraiment de notre gisement ou bien d'un autre qui se trouve à peu de distance ?

Le petit site minier d'Erlenbach permet d'observer en abondance des espèces minérales formées dans les parties altérées d'une lentille de minerai $\mathrm{Pb}-\mathrm{Zn}$ sulfuré, ce qui n'est plus si fréquent aujourd'hui dans cette ancienne région minière. À côté de la smithsonite (calamine) très abondante mais généralement peu esthétique, on y recense une vingtaine d'espèces minérales (Tableau 3) dont deux espèces sont nouvelles pour la Belgique : la coronadite et le minium. Un alumino-silicate de zinc apparemment non encore décrit y est également observé, mais la petitesse des cristallisations ne nous a permis de le décrire.

La minéralogie de ce site, bien qu'apparemment moins riche que celle de Rabotrath situé sur la même commune de Lontzen (Blondieau et al., 2016), permet néanmoins de dégager quelques caractéristiques régionales.

Nous pouvons notamment souligner que :

- La smithsonite est le minerai calaminaire prépondérant de ce district minier. L'hydrozincite et l'hémimorphite ne sont, apparemment, que des espèces accessoires. L'hémimorphite semble toutefois avoir été observée à Erlenbach mais nous n'avons jamais pu l'identifier dans le matériel que nous avons prélevé. La willémite n'a été observée ni à Rabotrath, ni à Erlenbach alors qu'elle semble avoir été abondante à La Calamine (Altenberg) mais aussi à Schimper sur le filon de Bleyberg. (Blondieau \& Polrot, 2011). 
Bulletin de la Société des Sciences de Liège, Vol. 87, articles, 2018, p. 22 - 55

- La chalcophanite semble fréquente en association avec la smithsonite.

- L'allophane et la dundasite sont des espèces fragiles et discrètes mais semblent apparemment faire partie des associations régionales observées.

- La plumbojarosite est souvent observée sur des rognons de galène.

- La minéralogie de ce vieux district minier semble riche en espèces minérales et il est fort probable que d'autres espèces y seront encore découvertes à l'occasion d'études nouvelles de l'un ou l'autre de ces gisements.

Tableau 3 : Espèces minérales d'Erlenbach. Les espèces en "gras » sont nouvelles pour la Belgique.

\begin{tabular}{|c|c|}
\hline Minéral & Formule chimique idéale \\
\hline Allophane & $\mathrm{Al}_{2} \mathrm{O}_{3}\left(\mathrm{SiO}_{2}\right)_{1.3-2.0} \cdot 2.5-3.0 \mathrm{H}_{2} \mathrm{O}$ \\
\hline Anglésite & $\mathrm{PbSO}_{4}$ \\
\hline Barite & $\mathrm{BaSO}_{4}$ \\
\hline Cérusite & $\mathrm{PbCO}_{3}$ \\
\hline Chalcophanite & $\mathrm{ZnMn}_{3}^{4+} \mathrm{O}_{7} \cdot 3 \mathrm{H}_{2} \mathrm{O}$ \\
\hline Coronadite & $\mathrm{Pb}\left(\mathrm{Mn}^{4+}{ }_{6} \mathrm{Mn}^{3+}{ }_{2}\right) \mathrm{O}_{16}$ \\
\hline Covellite & $\mathrm{CuS}$ \\
\hline Dundasite & $\mathrm{PbAl}_{2}\left(\mathrm{CO}_{3}\right)_{2}(\mathrm{OH})_{4} \cdot \mathrm{H}_{2} \mathrm{O}$ \\
\hline Galène & $\mathrm{PbS}$ \\
\hline Goethite & $\mathrm{FeO}(\mathrm{OH})$ \\
\hline Hématite & $\mathrm{Fe}_{2} \mathrm{O}_{3}$ \\
\hline Hetaerolite & $\mathrm{ZnMn}_{2}^{3+}{ }_{2} \mathrm{O}_{4}$ \\
\hline Hydrohetaerolite & $\mathrm{HZnMn}^{3+}{ }_{1.7} \mathrm{O}_{4}$ \\
\hline Hydrozincite & $\mathrm{Zn}_{5}\left(\mathrm{CO}_{3}\right)_{2}(\mathrm{OH})_{6}$ \\
\hline Kaolinite & $\mathrm{Al}_{2} \mathrm{Si}_{2} \mathrm{O}_{5}(\mathrm{OH})_{4}$ \\
\hline Malachite & $\mathrm{Cu}_{2} \mathrm{CO}_{3}(\mathrm{OH})_{2}$ \\
\hline Marcasite & $\mathrm{FeS}_{2}$ \\
\hline Minium & $\mathbf{P b}^{2+} \mathbf{P b}^{4+} \mathbf{O}_{4}$ \\
\hline Plumbojarosite & $\mathrm{Pb}_{0,5} \mathrm{Fe}_{3}\left(\mathrm{SO}_{4}\right)_{2}(\mathrm{OH})_{6}$ \\
\hline Pyromorphite & $\mathrm{Pb}_{5}\left(\mathrm{PO}_{4}\right)_{3} \mathrm{Cl}$ \\
\hline Quartz & $\mathrm{SiO}_{2}$ \\
\hline Smithsonite & $\mathrm{ZnCO}_{3}$ \\
\hline Soufre & $\mathrm{S}$ \\
\hline Sphalérite & $(\mathrm{Zn}, \mathrm{Fe}) \mathrm{S}$ \\
\hline Non identifié & Al-Si-Zn-O \\
\hline
\end{tabular}

\section{$\underline{\text { Remerciements : }}$}

Nous souhaitons remercier Fabrice Dal Bo pour l'aide importante apportée lors de l'utilisation des diffractomètres au laboratoire et Charles Grootenclaes pour les renseignements concernant Lontzen. 
Nos remerciements vont aussi à Pascal Chollet qui a réalisé de magnifiques photos de nos très petits minéraux, à Philippe Compère pour son aide lors de l'utilisation du microscope électronique à balayage ainsi qu'à Marie Meunier pour les photos du site.

Enfin, on peut également saluer la gentillesse des exploitants de la ferme d'Erlenbach qui nous ont toujours autorisés à échantillonner sur le site.

\section{Références}

Blondieau, M. \& Polrot, F., 2011. Les travaux miniers de Schimper, siège sud de la mine du Bleyberg (Plombières, Belgique) : plomb, zinc mais aussi argent. Geological Survey of Belgium, Professional Paper, 310, $57 \mathrm{p}$.

Blondieau, M., Puccio S., Compère, P. et Hatert, F., 2016. Minéralogie de l'ancienne mine plomb-zinc de Rabotrath (Lontzen, Belgique). Bulletin de la Société Royale des Sciences de Liège, 85, 30-55.

Blondieau, M., Puccio S et Hatert, F., 2017. Minéralogie de l'ancienne mine plomb de Masbourg (Nassogne, Province de Luxembourg, Belgique). Bulletin de la Société Royale des Sciences de Liège, 86, 169-201.

Dejonghe, L. \& Jans, D., 1983. Les gisements plombo-zincifères de l'Est de la Belgique. Chron. Rech. Min. 473, 3-24.

Dejonghe, L., Ladeuze, F. \& Jans, D., 1993. Atlas des gisements plombo-zincifères du synclinorium de Verviers (est de la Belgique). Mémoires pour servir à l'explication des Cartes Géologiques et Minières de la Belgique, 33, 483 p.

Dejonghe, L. \& Ladeuze, F., 1994. Schmalgraf : un gisement plombo-zincifère dans le calcaire carbonifère de l'Est de la Belgique. Bulletin de la Société Belge de Géologie, 103 (1-2), 135-147.

Favreau, G., 2006. Faces version 4.4, logiciel interactif de tracé de cristaux. Editions Favreau, 1995-2006.

Fransolet, A.-M. \& Mélon, J., 1975. Données nouvelles sur des minéraux de Belgique. Bulletin de la Société Royale des Sciences de Liège, 44, 157.

Hatert, F., Deliens, M., Fransolet, A.-M. \& Van Der Meersche, E., 2002. Les minéraux de Belgique, deuxième édition, $304 \mathrm{p}$.

Kolitsch, U., Rieck, B., Brandstätter, F., Schreiber, F., Fabritz, K. H., Blass, G. \& Gröbner, J., 2014. Neufunde au dem alten Bergbau und den Schlacken von Laurion (I). Mineralien Welt 25 (1), 60-75.

Lebocey, J., 2009. La galène de Planioles, Lot. Le Règne Minéral, 89, 5-12.

Mindat, 2017. Page "Erlenbach" : https://www.mindat.org/loc-249528.html [consultation en Février 2017].

Polrot, F., 2002 - Inventaire des traces liées à d'anciennes industries extractives sur les carbonates du paléozoïque de 1'Est de la Belgique. Professional paper, Service Géologique de Belgique, 296, 339 pages.

Union Minière, 2000. Renonciation à la concession de la Vieille-Montagne. Document inédit. 